\title{
The Kervaire invariant of immersions
}

\author{
R.L. Cohen ${ }^{1}$, J.D.S. Jones ${ }^{2}$, and M.E.Mahowald ${ }^{3}$ \\ ${ }^{1}$ Department of Mathematics, Stanford University, Stanford, CA 94305, USA \\ 2 Department of Mathematics, University of Warwick, Coventry, England \\ ${ }^{3}$ Department of Mathematics, Northwestern University, Evanston, IL 60201, USA
}

The purpose of this paper is to study and compute E. Brown's generalized Kervaire invariant [5]

$$
K: \Omega_{*}^{G} \rightarrow \mathbb{Z} / 8
$$

where $\Omega_{*}^{G}$ is an appropriate cobordism theory of immersed submanifolds of Euclidean space. By generalizing techniques of Browder [2] we shall give necessary and sufficient Adams spectral sequence conditions for an element in $\Omega_{*}^{G}$ to have nonzero Kervaire invariant. Also we will prove that for every $j \geqq 1$ there exists a closed, differentiable manifold of dimension $2^{j+1}-2$, together with an immersion in $\mathbb{R}^{2^{j+1}}$ which has nonzero Kervaire invariant. Before we state our results more precisely, we first recall some preliminaries about the Kervaire invariant.

The classical Kervaire invariant of stably framed corbodism, $K$ : $\Omega_{4 k+2}^{\mathrm{fr}} \rightarrow \mathbb{Z} / 2$, is the obstruction to a framed cobordism class containing a framed homotopy sphere. The question of the existence of smooth, stably framed, closed manifolds with nonzero Kervaire invariant has intrigued topologists since the early 1960 's.

By using the Thom-Pontrjagin construction which equates framed cobor$\operatorname{dism}, \Omega_{*}^{\mathrm{fr}}$, with the stable homotopy groups of spheres, $\pi_{*}^{s}\left(S^{0}\right)$, W. Browder gave necessary and sufficient conditions on the mod 2 Adams spectral sequence for there to exist elements in $\Omega_{*}^{\mathrm{fr}}=\pi_{*}^{s}\left(S^{0}\right)$ which have Kervaire invariant one. In [2] he proved the following.

Theorem. The Kervaire invariant $K: \Omega_{2 n}^{\mathrm{fr}} \rightarrow \mathbb{Z} / 2$ is zero unless $n$ is of the form $2^{j}$ -1 . Furthermore, an element $\theta_{j} \in \Omega_{2 j+1-2}^{\mathrm{fr}_{\mathrm{r}}}=\pi_{2^{j+1}-2}^{\mathrm{s}}\left(S^{0}\right)$ has Kervaire invariant one if and only if it is represented by $h_{j} h_{j} \in \mathrm{Ext}_{A}(\mathbb{Z} / 2, \mathbb{Z} / 2)$ in the $E_{2}$ term of the Adams spectral sequence.

During the preparation of this work the first and third authors were supported by NSF grants, and the first author by an A.P. Sloan foundation fellowship. The first author would also like to thank the mathematics department at Princeton University for its hospitality during the time the work was completed 
In this theorem, $A$ is the mod 2 Steenrod algebra and $h_{j} \in \mathrm{Ext}_{A}(\mathbb{Z} / 2, \mathbb{Z} / 2)$ is the generator in bidegree $\left(1,2^{j}\right)$. By work of Mahowald and Tangora and of Barratt, Jones, and Mahowald (see $[21,1]$ ) an element $\theta_{j} \in \pi_{2^{j+1}-2}^{s}\left(S^{0}\right)$ with the required properties is known to exist for $j \leqq 5$.

As observed by Browder [2], the essential ingredient contained in a framed $2 n$-dimensional manifold $\left(M^{2 n}, f\right)$ that allows one to define its Kervaire invariant, is a trivialization of the $(n+1)^{\text {st }} \mathrm{Wu}$ class, $v_{n+1}$, of the stable normal bundle. This idea was carried further by Brown in $[4,5]$ when he described how to define a $\mathbb{Z} / 8$-valued Kerviare invariant

$$
K: \Omega_{2 n}^{\xi} \rightarrow \mathbb{Z} / 8
$$

where $\Omega_{*}^{\xi}$ is any cobordism theory with vanishing $(n+1)^{\text {st }}$ Wu class, $v_{n+1}(\xi)$.

Now let $G$ be one of the Lie groups $O(1)$ or $U(1)=S O(2)$, and let $\Omega_{*}^{G}$ denote the cobordism group of manifolds together with a reduction of the structure group of their stable normal bundles from the infinite orthogonal group $O$ to $G$. By work of M. Hirsch [13] $\Omega_{*}^{o(1)}$ can be thought of as cobordism classes of manifolds immersed in codimension one Euclidean space. Similarly $\Omega_{*}^{S O(2)}$ =cobordism classes of oriented manifolds immersed in codimension 2 Euclidean space.

Notice that $\Omega_{*}^{\text {fr }}$ can also be thought of as an immersion-theoretic cobordism theory since $S O(1)=$ the trivial group, $\Omega_{*}^{\mathrm{fr}}=\Omega_{*}^{S O(1)}=$ the cobordism group of oriented manifolds immersed in codimension one Euclidean space.

An easy calculation shows that

$$
0=v_{n+1} \in H^{n+1}(B G ; \mathbb{Z} / 2) \quad \text { if } \begin{cases}n \neq 2^{k}-2 & \text { if } G=O(1) \\ n \neq 2^{k}-3 & \text { if } G=S O(2)\end{cases}
$$

and therefore we have a Brown-Kervaire invariant

$$
K: \Omega_{2 n}^{G} \rightarrow \mathbb{Z} / 8
$$

for $n$ as above. Our first result is a generalization of Browder's theorem, both in its statement and its proof.

Theorem. Unless $n=1$ or 3 and $G=O(1)$, the Kervaire invariant of a class $x \in \Omega_{2 n}^{G}=\pi_{2 n}^{s}(M G)$ is nonzero if and only if $x$ is represented in the Adams spectral sequence by a class in $\operatorname{Ext}_{A}^{2}\left(H^{*}(M G), \mathbb{Z} / 2\right)$ in the image of multiplication by $h_{j} h_{j}$ under the Yoneda pairing

$$
\operatorname{Ext}_{A}^{0}\left(H^{*}(M G), \mathbb{Z} / 2\right) \otimes \operatorname{Ext}_{A}^{2}\left(\mathbb{Z}_{2}, \mathbb{Z}_{2}\right) \rightarrow \operatorname{Ext}_{A}^{2}\left(H^{*}(M G) ; \mathbb{Z}_{2}\right) .
$$

Here $M G$ is the Thom spectrum of the universal $G$-vector bundle over $B G$.

We will actually significantly cut down the list of classes in $\operatorname{Ext}_{A}^{2}\left(H^{*}(M G), \mathbb{Z} / 2\right)$ that can represent cobordism classes with nonzero Kervaire invariant by showing that many of the classes occurring in this theorem carry nonzero differentials in the Adams spectral sequence. A more precise, stronger version of this theorem is stated in Sect. 1 (Theorems (1.6) and (1.8)).

Our second main result is the construction of classes in $\Omega_{*}^{S O(2)}$ having nonzero Kervaire invariant. These are constructed using techniques of Mahowald [19]. More specifically we will prove the following. 
Theorem. Let $u_{*}: \operatorname{Ext}_{A}\left(\mathbb{Z}_{2}, \mathbb{Z}_{2}\right) \rightarrow \operatorname{Ext}_{A}\left(H^{*}(M S O(2)), \mathbb{Z} / 2\right)$ be the homomorphism induced by the Thom class, $u: S^{0} \rightarrow M S O(2)$. Then for every $j \geqq 1$ there exists an element $\left.\theta_{j} S O(2)\right) \in \Omega_{2^{j+1}-2}^{S O(2)} \cong \pi_{2 j+1-2}^{s}(M S O(2))$ which is represented by $u_{*}\left(h_{j} h_{j}\right)$ in the Adams spectral sequence. In particular $\theta_{j}(S O(2))$ has nonzero Kervaire invariant.

We remark that this result can be viewed as in some sense the second best possible solution to the Kervaire invariant problem in the oriented immersion setting, since $\Omega_{*}^{\mathrm{fr}}=\Omega_{*}^{S O(1)}$.

As we shall see, a direct corollary of this theorem is a recent result of $\mathrm{R}$. Bruner [8] stating that $h_{2} h_{j}^{2} \in \mathrm{Ext}_{A}^{3}(\mathbb{Z} / 2, \mathbb{Z} / 2)$ is an infinite cycle in the Adams spectral sequence for the homotopy groups of spheres. Also, our techniques will yield a simplified proof of Mahowald's theorem [19] that $h_{1} h_{j} \in \mathrm{Ext}_{A}$ $\cdot(\mathbb{Z} / 2, \mathbb{Z} / 2)$ is an infinite cycle.

This paper is organized as follows. In Sect. 1 we describe a generalization of Brown's Kervaire invariant and state our results. In Sect. 2 we use Browder's techniques to describe a secondary cohomology operation that detects the Kervaire invariant. In Sect. 3 we apply this operation in the case of $\Omega_{*}^{G}$ and prove Theorems (1.6) and (1.8). The construction of the classes $\theta_{j}(S O(2))$ having nonzero Kervaire invariant is done in Sect. 4. Certain Witt group calculations referred to in Sect. 1 are done in the appendix.

We remark that the results of this paper solve problems described by Browder in [3].

Throughout the rest of this paper all (co)-homology will be taken with $\mathbb{Z} / 2$ coefficients, unless otherwise indicated.

\section{$\S 1$. Brown's Kervaire invariant and the statement of results}

Let $B$ be a space and $\xi: B \rightarrow B O$ represent a stable vector bundle over $B$. Let $T(\xi)$ be the associated Thom spectrum, normalized so that the inclusion of the fibre determines an element of $\pi_{0}^{s}(T(\xi))$. Let $M$ be a manifold with stable normal bundle $v(M)$.

(1.1) Definition. $A$-orientation for $M$ is a map $\Phi: M \rightarrow B$ so that the pullback bundle, $\Phi^{*}(\xi)$, is isomorphic to $v(M)$.

Let $\Omega_{*}^{\xi}(-)$ denote the bordism theory of $\xi$-oriented manifolds. Thus for any space $X \Omega_{n}^{\xi}(X)$ consists of bordism classes of triples $(M, \Phi ; f)$, where $M$ is a closed $n$-manifold, $\Phi$ is a $\xi$-orientation of $M$, and $f: M \rightarrow X$ is a map. Write $[M, \Phi ; f]$ for the associated bordism class. Recall that the Thom-Pontryagin construction gives isomorphisms

$$
\Omega_{*}^{\xi}(X) \cong \pi_{*}^{s}\left(X_{+} \wedge T(\xi)\right)
$$

and

$$
\tilde{\Omega}_{*}^{\xi}(X) \cong \pi_{*}^{s}(X \wedge T(\xi)) .
$$

In the first isomorphism $X_{+}$denotes $X$ with a disjoint basepoint. 
Let $K_{n}$ be an Eilenberg-MacLane space of type $(\mathbb{Z} / 2, n)$, and let $(M, \Phi)$ be a $\xi$-oriented $2 n$-dimensional manifold. In [5] Brown studies the function

$$
q: H^{n}\left(M^{2 n}\right) \rightarrow \tilde{\Omega}_{2 n}^{\xi}\left(K_{n}\right)
$$

defined as follows: Regard $\alpha \in H^{n}\left(M^{2 n}\right)$ as a map $\alpha: M \rightarrow K_{n}$, then

$$
q(\alpha)=[M, \Phi ; \alpha]-[M, \Phi] \in \tilde{\Omega}_{2 n}\left(K_{n}\right) .
$$

Brown studied the group $\tilde{\Omega}_{2 n}^{\xi}\left(K_{n}\right)$ by considering the function

$$
P: \tilde{\Omega}_{2 n}^{\xi}\left(K_{n}\right) \rightarrow H_{n} B
$$

defined by $P[M, \Phi ; \alpha]=\Phi_{*}([M] \cap \alpha)$. Here $\alpha \in H^{n}(M),[M] \in H_{2 n}(M)$ is the fundamental class, and " $\cap$ " denotes the cap product operation. In [5] Brown proved the following:

(1.2) Theorem. a) Suppose the $(n+1)$-st $W u$ characteristic class, $v_{n+1}(\xi)$ is nonzero. Then the map $P: \tilde{\Omega}_{2 n}^{\xi}\left(K_{n}\right) \rightarrow H_{n}(B)$ is an isomorphism, and the map $q$ : $H^{n}(M) \rightarrow \tilde{\Omega}_{2 n}^{\xi}\left(K_{n}\right)$ is a group homomorphism.

b) If $v_{n+1}(\xi)=0$. then there is an exact sequence

$$
0 \rightarrow \mathbb{Z} / 2 \stackrel{i}{\longrightarrow} \tilde{\Omega}_{2 n}^{\xi}\left(K_{n}\right) \stackrel{P}{\longrightarrow} H_{n} B \rightarrow 0 .
$$

In this case $q: H^{n} M \rightarrow \tilde{\Omega}_{2 n}^{\xi}\left(K_{n}\right)$ is quadratic; that is, $q(\alpha+\beta)=q(\alpha)+q(\beta)$ $+i(\alpha \cdot \beta)$, where $\alpha \cdot \beta \in \mathbb{Z} / 2$ is the mod 2 intersection number of $\alpha$ and $\beta$. Furthermore, this exact sequence is split if and only if $v_{n}(\xi)=0$.

In the case when $v_{n+1}(\xi)=0$, the Kervaire invariant of a $\xi$-oriented manifold $\left(M^{2 n}, \Phi\right)$ is defined to be the Witt group classification of the quadratic form $q$. Recall that this classification works as follows:

Let $A$ be an abelian group and suppose $i: \mathbb{Z} / 2 \hookrightarrow A$ is an injection. Let $V$ be a finite dimensional $\mathbb{Z} / 2$ vector space equipped with a nonsingular bilinear pairing

$$
\langle,\rangle: V \times V \rightarrow \mathbb{Z} / 2 \text {. }
$$

A function $q: V \rightarrow A$ is said to be quadratic if it satisfies $q(v+w)=q(v)+q(w)$ $+i\langle v, w\rangle$. The form $(V, q)$ is said to be Witt equivalent to zero if there is a subspace $Q$ of $V$ with 2 rank $Q=$ rank $V$ and $q(v)=0$ for all $v \in Q$. Two forms $\left(V_{1}, q_{1}\right)$ and $\left(V_{2}, q_{2}\right)$ are said to be Witt equivalent if the form $\left(V_{1} \oplus V_{2}, q_{1}-q_{2}\right)$ is Witt equivalent to zero. The Witt group of $A, W(A)$ is defined to be the set of Witt equivalence classes of such forms. Addition is induced by direct sum. If $(V, q)$ is a form, let $[V, q]$ be its Witt class in $W(A)$.

From here on we restrict to the case when $\xi$ is a stable vector bundle with $v_{n+1}(\xi)=0$.

Let $W_{2 n}^{\xi}$ denote the Witt group $W\left(\tilde{\Omega}_{2 n}^{\xi}\left(K_{n}\right)\right)$. We define the Kervaire invariant homomorphism

$$
K: \Omega_{2 n}^{\xi} \rightarrow W_{2 n}^{\xi}
$$


by the rule $K\left[M^{2 n}, \Phi\right]=\left[H^{n}(M), q\right] \in W_{2 n}^{\xi}$, where $q$ is the quadratic form described above.

To see that $K$ is well defined, observe that if there exists a $(2 n+1)$ dimensional $\xi$-oriented manifold $(W, \Psi)$ with $\partial(W, \Psi)=(M, \Phi)$, then $q$ : $H^{n}(M) \rightarrow \tilde{\Omega}_{2 n}^{\xi}\left(K_{n}\right)$ vanishes on $\mathrm{Q}=\operatorname{Image}\left(H^{n} W \rightarrow H^{n} M\right)$. A standard Poincare duality argument shows that $2 \operatorname{rank} Q=\operatorname{rank} H^{n} M$. Therefore the Witt class $\left[H^{n} M, q\right]$ is zero.

We now briefly discuss some algebraic properties of certain Witt groups $W(A)$. Proofs will be supplied in the appendix. Observe first that if $A=\tilde{\Omega}_{2 n}^{\xi}\left(K_{n}\right)$, then by $(1.2 \mathrm{~b}) 4 A=0$. In what follows we shall assume that $A$ is an abelian group with $4 A=0$.

Let $(V, q)$ be an $A$-valued quadratic form, and let $h: A \rightarrow \mathbb{Z} / 4$ be a homomorphism with the property that the composition $h i: \mathbb{Z} / 2 \rightarrow A \rightarrow \mathbb{Z} / 4$ is injective. Then the composite $q_{h}=h q: V \rightarrow \mathbb{Z} / 4$ is also quadratic. In $[4,5]$ Brown constructed an isomorphism $\sigma: W(\mathbb{Z} / 4) \rightarrow \mathbb{Z} / 8$. Define $\sigma_{h}(V, q)=\sigma(V, h q)$.

(1.3) Lemma. Let $\oplus_{h} \mathbb{Z} / 8$ be a direct sum of copies of $\mathbb{Z} / 8$ indexed by all homomorphisms $h: A \rightarrow \mathbb{Z} / 4$ with $i h: \mathbb{Z} / 2 \rightarrow \mathbb{Z} / 4$ injective. Then the homomorphism $\sigma=\oplus \sigma_{h}: W(A) \rightarrow \underset{h}{\oplus} \mathbb{Z} / 8$ is injective.

Now suppose $h_{1}, h_{2}: A \rightarrow \mathbb{Z} / 4$ are two homomorphisms such that $i h_{1}$ and $i h_{2}$ are both injective. Then $h_{1} q-h_{2} q: V \rightarrow \mathbb{Z} / 4$ is a homomorphism so it is given by $x \rightarrow j\langle d, x\rangle$ for some $d \in V$, where here, and for the rest of the paper we let $j: \mathbb{Z} / n \subset \mathbb{Z} / 2 n$ be the obvious inclusion. An elementary argument using Brown's invariant $\sigma$ proves the following:

(1.4) Lemma. $\sigma_{h_{1}}(V, q)-\sigma_{h_{2}}(V, q)=j q_{h_{1}}(d) \in \mathbb{Z} / 8$.

In the case when $A=\tilde{\Omega}_{2 n}^{\xi}\left(K_{n}\right)$, we write $K_{h}(M ; \Phi)$ for the $\mathbb{Z} / 8$-valued invariant determined by $h: A \rightarrow \mathbb{Z} / 4$. If $h_{1}$ and $h_{2}$ are two such homomorphisms, then in view of (1.2) part b we have that $h_{1}-h_{2}=f P$ where $f \in H^{n} B$. In view of (1.4) we may conclude the following:

(1.5) Lemma. $K_{h_{1}}(M, \Phi)-K_{h_{2}}(M ; \Phi)=j q_{h_{1}}\left(\Phi^{*} f\right)$.

Recall that $W(\mathbb{Z} / 2)=\mathbb{Z} / 2$ and the Arf invariant of a quadratic form determines its Witt class. In cobordism theory this reappears as the case $B$ $=$ point, $\xi$ is therefore trivial, and $\Omega_{*}^{\xi}$ is framed cobordism $\tilde{\Omega}_{*}^{\mathrm{fr}}$. Then $\Omega_{*}^{\mathrm{fr}} K_{n}$ $=\tilde{\pi}_{2 n}^{s} K_{n}=\mathbb{Z} / 2$ and we recover the classical Kervaire invariant $K: \Omega_{2 n}^{\mathrm{fr}} \rightarrow \mathbb{Z} / 2$ [17].

We now come to the main results of the paper. The prototype for our theorems is Browder's result [2] in which he reduces the calculation of $K: \tilde{\Omega}_{2 n}^{\mathrm{fr}}$ $\cong \pi_{2 n}^{s}\left(S^{0}\right) \rightarrow \mathbb{Z} / 2$ to an Adams spectral sequence question. In order to state our results we first adopt some notation. If $X$ is a spectrum, let $\operatorname{Ext}(X)$ denote the group $\operatorname{Ext}_{A}\left(H^{*}(X), \mathbb{Z} / 2\right)$, where $A$ is the $\bmod 2$ Steenrod algebra. Recall that $\operatorname{Ext}(X)$ is the $E_{2}$ term of the Adams spectral sequence converging to the 2primary part of $\pi_{*}^{s}(X)$. Recall also that $\operatorname{Ext}(X)$ is a module over the algebra 
$\operatorname{Ext}\left(S^{0}\right)$, and that

$$
\operatorname{Ext}^{1, t}\left(S^{0}\right)=\left\{\begin{array}{cl}
\mathbb{Z} / 2 & \text { generated by a class } h_{i} \text { if } t=2^{i} \\
0 & \text { otherwise. }
\end{array}\right.
$$

Our first result concerns the case $B=B O(1)=R P^{\infty}$, and $\xi=\gamma$, the universal line bundle. Then $\Omega_{*}^{\xi}=\Omega_{*}^{O(1)}$ is the cobordism group of codimension one immersions, as observed in the introduction. An easy calculation gives that the Wu classes $v_{q}(\gamma)=0$ if and only if $q \neq 2^{k}-1$, and we therefore get a Kervaire invariant for $2 n$-dimensional manifolds for $2 n \neq 2^{k+1}-4$. From 1.2 we read off the bordism groups

$$
\tilde{\Omega}_{2 n}^{o(1)}\left(K_{n}\right)= \begin{cases}\mathbb{Z} / 2 & \text { if } n=2^{k}-2 \\ \mathbb{Z} / 4 & \text { if } n=2^{k}-1 \\ \mathbb{Z} / 2 \oplus \mathbb{Z} / 2 & \text { if } n \neq 2^{k}-1,2^{k}-2\end{cases}
$$

From now on we exclude the cases $n=2^{k}-2$, since the Kervaire invariant is not defined in these dimensions. From the appendix we can read off the Witt groups

$$
W_{2 n}^{O(1)}=\left\{\begin{array}{cl}
\mathbb{Z} / 2 \oplus \mathbb{Z} / 2 & \text { if } n \neq 2^{k}-1 \\
\mathbb{Z} / 8 & \text { if } n=2^{k}-1
\end{array}\right.
$$

(1.6) Theorem. a) $K: \Omega_{2 n}^{O(1)} \rightarrow W_{2 n}^{O(1)}$ is zero if $2 n \neq 2^{k+1}+2^{m}-4$.

b) $K: \Omega_{2}^{O(1)} \rightarrow \mathbb{Z} / 8$ is an isomorphism.

c) $K: \Omega_{6}^{O(1)} \rightarrow \mathbb{Z} / 8$ is surjective.

d) If $2 n \neq 2,6$, then $2 K(x)=0$ for all $x \in \Omega_{2 n}^{O(1)}$.

e) If $2 n=2^{k+1}+2^{m}-4$ but $2 n \neq 2,6$, then $x \in \tilde{\Omega}_{2 n}^{O(1)}=\pi_{2 n}^{s}(M O(1))$ has nonzero Kervaire invariant if and only if $x$ is represented by a class of the form $e_{2^{m}-2} h_{k} h_{k}$ for $m \leqq 3$ or $k \leqq 2, e_{2^{k}-2} h_{k} h_{k}$, or $e_{2^{k+1}-2} h_{k} h_{k} \in \operatorname{Ext}^{2}(M O(1))$ in the Adams spectral sequence.

Here $e_{q} \in H_{q}(M O(1))=H_{q+1}\left(R P^{\infty}\right)$ is the generator. It determines an element in $\mathrm{Ext}^{0, q}(M O(1))$ if and only if $q=2^{r}-2$.

Remarks. 1. The qualitative statement of this theorem is the same as that of Browder's [2]. Namely, unless $n=1,3$, the only elements in $\tilde{\Omega}_{2 n}^{O(1)}$ that have nonzero Kervaire invariant are those elements in $\pi_{2 n}^{s}(M O(1))$ detected by classes in $\operatorname{Ext}^{2}(M O(1))$ in the image of multiplication by $h_{j} h_{j}$ under the pairing

$$
\operatorname{Ext}^{0}(M O(1)) \otimes \operatorname{Ext}^{2}\left(S^{0}\right) \rightarrow \operatorname{Ext}^{2}(M O(1)) .
$$

2. Let $x \in \tilde{\Omega}_{2 n}^{o(1)}$ be in the image of the natural map from framed cobordism, $\Omega_{*}^{\mathrm{fr}} \rightarrow \tilde{\Omega}_{*}^{O(1)}$. Then by Browder's theorem [2] $x$ has nonzero Kervaire invariant if and only if $x$ is represented by $e_{0} h_{j} h_{j} \in \operatorname{Ext}^{2}(M O(1))$ in the Adams spectral sequence.

Recall that in [12] Cohen and Mahowald showed that there exist elements

$$
\tilde{\eta}_{j} \in \pi_{2^{j}}^{s}\left(R P^{\infty}\right)=\pi_{2^{j-1}}^{s}(M O(1))
$$

detected by $e_{2^{j}-2} h_{1} \in \operatorname{Ext}^{1}(M O(1))$. Under the Kahn-Priddy map $\lambda: R P^{\infty} \rightarrow S^{0}$ it was shown that $\tilde{\eta}_{j}$ maps to $\eta_{j} \in \pi_{2 j}^{s}\left(S^{0}\right)$, the class constructed by Mahowald in [19] that is detected by $h_{1} h_{j} \in \operatorname{Ext}^{2}\left(S^{0}\right)$. 
Define $\rho_{j} \in \pi_{2,}^{s}(M O(1))$ to be the class

$$
\rho_{j}=\tilde{\eta}_{j} \wedge \eta: S^{2 J-1} \wedge S^{1} \rightarrow M O(1) \wedge S^{0}=M O(1)
$$

where $\eta \in \pi_{1}^{s}\left(S^{0}\right)=\mathbb{Z} / 2$ is the generator. Since $\eta$ is detected by $h_{1} \in \operatorname{Ext}^{1}\left(S^{0}\right), \rho_{j}$ is detected by $e_{2,-2} h_{1} h_{1} \in \operatorname{Ext}^{2}(M O(1))$. By (1.6) we then have the following.

(1.7) Corollary. For each $j>2$ the class $\rho_{j} \in \pi_{2 j}(M O(1))=\tilde{\Omega}_{2 j}^{o(1)}$ has nonzero Kervaire invariant.

Remark. The classes $\rho_{i} \in \tilde{\Omega}_{2^{j}}^{O(1)}$ are of only limited interest since as observed above they are not in the image of framed cobordism.

We now turn to the case $B=B S O(2)=B U(1)=\mathbb{C} P^{\infty}$, and $\xi=\gamma$, the canonical complex line bundle. Thus $\Omega_{*}^{\gamma}=\Omega_{*}^{S O(2)}$ is cobordism classes of oriented manifolds immersed in codimension 2 Euclidean space. In this case the Wu class $v_{q}(\gamma)=0$ if and only if $q \neq 2^{k}-2$ and we therefore get a Kervaire invariant in dimensions $2 n$ so long as $n$ is not of the form $2^{k}-3$. Again, we read off the cobordism groups from (1.2) and the Witt groups from the appendix:

$$
\begin{gathered}
\tilde{\Omega}_{2 n}^{S O(2)}=\mathbb{Z} / 2 \oplus \mathbb{Z} / 2, \\
W_{2 n}^{S O(2)}=\mathbb{Z} / 2 \oplus \mathbb{Z} / 2 .
\end{gathered}
$$

(1.8) Theorem. a) $K: \tilde{\Omega}_{2 n}^{S O(2)} \rightarrow \mathbb{Z} / 2 \oplus \mathbb{Z} / 2$ is zero if $2 n \neq 2^{k+1}+2^{m}-6$.

b) If $2 n=2^{k+1}+2^{m}-6, \quad x \in \tilde{\Omega}_{2 n}^{s O(2)}=\pi_{2 n}^{s} M S O(2)$ has nonzero Kervaire invariant if and only if $x$ is represented by a class of the form $e_{2^{m}-4} h_{k} h_{k}$ for $m$ $=2,3$ or $k \leqq 2, e_{2^{k}-4} h_{k} h_{k}$, or $e_{2^{k-1}-4} h_{k} h_{k} \in \operatorname{Ext}^{2}(M S O(2))$ in the Adams spectral sequence.

Here $e_{2 q} \in H_{2 q} M S O(2)=H_{2 q+2}\left(\mathbb{C} P^{\infty}\right)$ is the generator; it determines an element of $\mathrm{Ext}^{0,2 q}(M S O(2))$ if and only if $q=2^{r}-2$.

Remark. Let $x \in \tilde{\Omega}_{2 n}^{S O(2)}$ be in the image of the natural map from framed cobor$\operatorname{dism}, \tilde{\Omega}_{*}^{\mathrm{fr}}=\tilde{\Omega}_{*}^{S O(1)} \rightarrow \tilde{\Omega}_{*}^{\text {SO(2)}}$. Then by Browder's theorem $x$ has nonzero Kervaire invariant if and only if $x$ is represented by $\left.e_{0} h_{j} h_{j} \in \mathrm{Ext}^{2 *} M S O(2)\right)$ in the Adams spectral sequence. The main positive result of this paper is that these classes are in fact infinite cycles. That is, we shall prove the following.

(1.9) Theorem. For every $j \geqq 1$ there exists an element $\theta_{j}(S O(2)) \in \Omega_{2^{j+1}}^{S O(2)}{ }_{-2}$ which is represented by $e_{0} h_{j} h_{j} \in \mathrm{Ext}^{2}(M S O(2))$ in the Adams spectral sequence. Hence $\theta_{j}(S O(2))$ represents a $2^{j+1}-2$ dimensional, $S O(2)$-oriented manifold having nonzero Kervaire invariant.

\section{§2. Detecting the Kervaire invariant}

In this section we use techniques of Browder to detect the Kervaire invariant.

As in the last section let $(M, \Phi)$ be a $\xi$-oriented $2 n$-dimensional manifold. Choose a homomorphism $h: \tilde{\Omega}_{2 n}^{\xi}\left(K_{n}\right) \rightarrow \mathbb{Z} / 4$. Let $v_{n}=v_{n}(M) \in H^{n} M$ be the $n$th $\mathrm{Wu}$ class. The following result is an easy consequence of the results in the appendix on the invariant $\sigma_{h}: W_{2 n}^{\xi} \rightarrow W(\mathbb{Z} / 4) \underset{\sigma}{\stackrel{\cong}{\rightarrow}} \mathbb{Z} / 8$ (cf. [4]). 
(2.1) Lemma. a) $K_{h}(M ; \Phi) \equiv \operatorname{rank} H^{n}(M) \equiv\left\langle v_{n}^{2} ;[M]\right\rangle \bmod 2$.

b) $K_{h}(M ; \Phi) \equiv q_{h}\left(v_{n}\right) \bmod 4$.

Notice that $v_{n}(M)=\Phi^{*} v_{n}(\xi)$ and in view of Lemma (1.5) it becomes interesting to compute $q_{h}\left(\Phi^{*}(x)\right)$ or better yet $q\left(\Phi^{*}(x)\right) \in \tilde{\Omega}_{2 n}^{\xi}\left(K_{n}\right)$.

(2.2) Lemma. If $x \in H^{n} B$, then $P q\left(\Phi^{*}(x)\right)=\Phi_{*}[M] \cap x \in H_{n} B$, where $P$ : $\tilde{\Omega}_{2 n}^{\Psi}\left(K_{n}\right) \rightarrow H_{n} B$ is the homomorphism of Theorem (1.2).

Proof. This is an easy exercise with the definitions and we leave it to the reader.

We will have considerable use for functional cohomology operations defined using the operation

$$
\sum_{i=0}^{n} S q^{n+1-i}: \bigoplus_{i} H^{i+k}(X) \rightarrow H^{n+1+k}(X)
$$

and a map $f: Y \rightarrow X$. The functional operation is defined on those classes $\left(x_{0}, \ldots, x_{n}\right) \in \bigoplus_{i=0}^{n} H^{i+k}(X)$ such that $\sum S q^{n+1-i}\left(x_{i}\right)=0$, and $f^{*}\left(x_{i}\right)=0$ for all $i$. The value of the functional operation is denoted by $\left(\sum_{i=0}^{n} S q^{n+1-i}\right)_{f}\left(x_{0}, \ldots, x_{n}\right)$ and lies in $H^{n+k}(Y) / \operatorname{Im}\left(f^{*}\right)$.

For any stable vector bundle $\zeta$ over $X$ let $\Psi_{\zeta}: H^{q}\left(X_{+}\right) \rightarrow H^{q}(T(\zeta))$ be the Thom isomorphism given by cup product with the Thom class. Finally, let $\alpha$ $=\alpha[M, \Phi] \in \pi_{2 n}^{\xi}(T(\xi))$ be the homotopy class corresponding to the cobordism class $[M ; \Phi]$.

We come now to the two main technical results of this section.

(2.3) Theorem. a) If $x \in H^{n} B$ then

$\sum_{i=0}^{n} S q^{n+1-i}\left(\Psi_{\xi}\left(x \cdot v_{i}(\xi)\right)\right)=0$.

b) Suppose $\Phi_{*}[M] \in H_{2 n} B$ is zero. Then, for all $x \in H^{n}(B), q\left(\Phi^{*}(x)\right)$ is in the image of $i: \mathbb{Z} / 2 \rightarrow \tilde{\Omega}_{2 n}^{\xi}\left(K_{n}\right)$, where $i$ is as in (1.2). Moreover, $q\left(\Phi^{*}(x)\right)$ is nonzero if and only if

$$
\left(\sum_{i=0}^{n} S q^{n+1-i}\right)_{a}\left(x_{0}, \ldots, x_{n}\right) \in H^{2 n} S^{2 n}
$$

is nonzero, where $x_{i}=\psi\left(x \cdot v_{i}(\xi)\right) \in H^{n+i}(T(\xi))$.

To state our next result we need some more notation. Let $H_{n}$ denote the Eilenberg-MacLane spectrum $H_{n}=\Sigma^{n} K \mathbb{Z}_{2}$. That is,

$$
\pi_{q} H_{n}=\left\{\begin{array}{cl}
\mathbb{Z} / 2 & \text { if } q=n \\
0 & \text { otherwise. }
\end{array}\right.
$$

Let $\tilde{H}_{0}$ denote the cofiber of the nontrivial map $\varepsilon: S^{0} \rightarrow H_{0}$. Notice that $H^{*} \tilde{H}_{0}=\tilde{A}$, the augmentation ideal of the Steenrod algebra $A$. Also observe that $H^{*}\left(\tilde{H}_{0} \wedge T(\xi)\right)=\tilde{A} \otimes H^{*}(T(\xi))$. 
Finally if $\gamma \in \pi_{*} X$ is a stable homotopy class, where $X$ is a spectrum, we write $A F(\gamma)=s$ to mean that $\gamma$ is represented in filtration $s$ in the Adams spectral sequence. In particular $A F(\gamma) \geqq 1$ means that $\gamma$ has zero Hurewicz image in $H_{*} X$, and $A F(\gamma) \geqq 2$ means that all functional cohomology operations defined using $\gamma$ vanish.

(2.4) Theorem. a) In $H^{*}\left(\bar{H}_{0} \wedge T(\xi)\right)$,

$$
\sum_{i=0}^{n} S q^{n+1-i}\left(\sum_{\substack{s+t=i \\
p+q=n+1}}\left(\begin{array}{c}
t+q \\
q
\end{array}\right) \chi\left(S q^{t+q}\right) \otimes \psi_{\xi}\left(v_{s}(\xi) v_{p}(\xi)\right)\right)=0
$$

where $\chi: A \rightarrow A$ is the canonical antiautomorphism.

b) If $\alpha \in \pi_{2 n}^{s}(T(\xi))$ and $A F(\alpha) \geqq 2$, then $\alpha$ has nonzero Kervaire invariant if and only if there is ane element $\beta \in \pi_{2 n+1}^{s}\left(\tilde{H}_{0} \wedge T(\xi)\right)$ such that $\partial_{*} \beta=\alpha$ and

$$
\left(\sum_{i=0}^{n} S q^{n+1=i}\right)_{\beta}\left(\sum_{\substack{s+t=i \\
p+q=n+1}}\left(\begin{array}{c}
t+q \\
q
\end{array}\right) \chi\left(S q^{t+q}\right) \otimes \psi_{\xi}\left(v_{s}(\xi) v_{p}(\xi)\right)\right) \neq 0 .
$$

Here $\partial_{*}: \pi_{k+1}\left(\tilde{H}_{0} \wedge T(\xi)\right) \rightarrow \pi_{k}(T(\xi))$ is the boundary homomorphism in the homotopy long exact sequence induced by the cofibration sequence

$$
T(\xi)=S^{0} \wedge T(\xi) \underset{\varepsilon \wedge 1}{\longrightarrow} H_{0} \wedge T(\xi) \rightarrow \tilde{H}_{0} \wedge T(\xi) .
$$

Our procedure for detecting the Kervaire invariant is contained in (2.1)(2.4). First we use characteristic numbers to determine the congruence of $K_{h}(M ; \Phi) \bmod 2$. Characteristic numbers also give some information on $K_{h}(M ; \Phi) \bmod 4$ and how $K_{h}(M ; \Phi)$ depends on the choice of homomorphism $h$. Next we assume that $\Phi_{*}[M]=0$, or equivalently, that $A F(\alpha) \geqq 1$. We then use $(2.3 \mathrm{~b})$ to determine $K_{h}(M ; \Phi) \bmod 4$ and to determine how $K_{h}(M, \Phi)$ depends on $h$. Finally we assume that $A F(\alpha) \geqq 2$ so that $K_{h}(M ; \Phi) \in\{0,4\} \subset \mathbb{Z} / 8$ and is independent of $h$. We then use $(2.4 \mathrm{~b})$ to compute $K_{h}(M ; \Phi)$.

We remark that our procedure has gaps in the most general setting. In particular it is possible that $\Phi_{*}[M] \neq 0$ but charcteristic numbers give no information concerning the Kervaire invariant. We will not pursue this possibility because as we shall see, this cannot happen in the cases in which we are most interested $\left(\Omega_{*}^{O(1)}\right.$ and $\left.\Omega_{*}^{S O(2)}\right)$.

We begin with the proofs of Theorems (2.3) and (2.4). First, we need an algebraic lemma, whose proof is contained in Papastavridis' paper [23]. Let $P$ and $Q$ be $A$-modules. Let $P \otimes Q$ have the diagonal $A$-module structure.

Lemma. If $x \in P$ and $y \in Q$ then

$$
x \otimes S q^{n+1}(y)=\sum_{i=0}^{n} S q^{n+1-i}\left(\chi\left(S q^{i}\right)(x) \otimes y\right) .
$$

The proof of this formula is given in [23]. It is a rather straightforward exercise using the defining relation for the antiautomorphism $\chi: \sum_{i=0}^{k} S q^{i} \chi\left(S q^{k-i}\right)$
$=0$. 
We next need a lemma from [2]. Define the spectrum $E_{n}$ to be the fiber of the map

$$
u_{\xi} \otimes S q^{n+1}: T(\xi) \wedge H_{n} \rightarrow H_{2 n+1} .
$$

where $u_{\xi} \in H^{0}(T(\xi))$ is the Thom class. The obvious map $T(\xi) \wedge K_{n} \rightarrow T(\xi) \wedge H_{n}$ lifts to $E_{n}$ since $S q^{n+1}$ acts trivially on the fundamental class $a_{n} \in H^{n}\left(K_{n}\right)$. We therefore have a homotopy commutative diagram

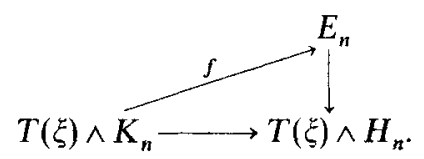

(2.6) Lemma. $f_{*}: \pi_{i}^{s}\left(T(\xi) \wedge K_{n}\right) \rightarrow \pi_{i} E_{n}$ is an isomorphism for $i \leqq 2 n$.

The proof of this is a direct cohomology calculation and is done in [2].

Before we proceed with the proof of Theorem(2.3) we need one more formula. By abuse of notation let $a_{n}$ denote both the nontrivial element in $H^{n} K_{n}$ and in $H^{n} H_{n}$. Recall that a $\mathbb{Z} / 2$-vector space basis for $H^{*} T(\xi) \otimes a_{n}$ is in turn a basis for the free $A$-module $H^{*}\left(T(\xi) \wedge H_{n}\right.$ ). Using (2.5) we can express the $k$-invariant, $u_{\xi} \otimes S q^{n+1}$, of $E_{n}$ in terms of this basis:

$$
\begin{aligned}
u_{\xi} \otimes S q^{n+1}\left(a_{n}\right) & =\sum_{i=0}^{n+1} S q^{n+1-i}\left(\chi\left(S q^{i}\right)\left(u_{\xi}\right) \otimes a_{n}\right) \\
& =\sum_{i=0}^{n} S q^{n+1-i}\left(\psi_{\xi}\left(v_{i}(\xi)\right) \otimes a_{n}\right) .
\end{aligned}
$$

The last equality holds because by definition of the $\mathrm{Wu}$ classes, $\chi\left(S q^{i}\right) u_{\xi}$ $=\psi_{\xi}\left(v_{i}(\xi)\right)$, and because $v_{n+1}(\xi)=0$.

Proof of Theorem (2.3). Let $x \in H^{n} B$, then since $S q^{n+1} x=0,0=u_{\xi} \otimes S q^{n+1} x$ in $H^{n}\left(T(\xi) \wedge B_{+}\right)$. Using formula (2.7) we get that

$$
0=\sum_{i=0}^{n} S q^{n+1-i}\left(\psi_{\xi}\left(v_{i}(\xi)\right) \otimes x\right) .
$$

Now let $\Delta: T(\xi) \rightarrow T(\xi) \wedge B_{+}$be the map of Thom spectra induced by the diagonal $B \rightarrow B \times B$. Using the fact that $\Delta^{*}\left(\psi_{\xi}(a) \otimes b\right)=\psi_{\xi}(a b)$ we can apply $\Delta^{*}$ to the above formula and get that

$$
\sum_{i=0}^{n} S q^{n+1-i}\left(\psi_{\xi}\left(x \cdot v_{i}(\xi)\right)\right)=0 .
$$

This is the statement of (2.3a).

To prove $(2.3 \mathrm{~b})$ assume that $\Phi_{*}[M]=0$; i.e. that $A F(\alpha) \geqq 1$. Let $\theta$ be the composition

$$
\theta: S^{2 n} \underset{\alpha}{\longrightarrow} T(\xi) \underset{\Delta}{\longrightarrow} T(\xi) \wedge B_{+} \underset{1 \wedge x}{\longrightarrow} T(\xi) \wedge K_{n}
$$

By definition the homotopy class of $\theta$ is just $q\left(\Phi^{*} x\right) \in \tilde{\Omega}_{2 n}^{\xi}\left(K_{n}\right)=\pi_{2 n}\left(T(\xi) \wedge K_{n}\right)$. Now since $\theta$ induces the zero homomorphism in homology the projection of $\theta$ 
to $\pi_{2 n}\left(T(\xi) \wedge H_{n}\right)$ is trivial. Thus by Lemma (2.6) and formula (2.7) we see that $\theta$ $=q\left(\Phi^{*}(x)\right)$ is nontrivial if and only if the functional operation

$$
\left(\sum_{i=0}^{n} S q^{n+1-i}\right)_{\theta}\left(\psi_{\xi}\left(v_{0}(\xi)\right) \otimes a_{n}, \psi_{\xi}\left(v_{1}(\xi)\right) \otimes a_{n}, \ldots, \psi_{\xi}\left(v_{n}(\xi)\right) \otimes a_{n}\right)
$$

is nonzero. Theorem $(2.3 \mathrm{~b})$ is now immediate.

Proof of Theorem (2.4) The strategy for our proof of (2.4) is the same as what we used for our proof of (2.3), but considerably more complicated. First, we need to recall a result of Mahowald [19].

Let $L$ be the double loop space of $S^{3}, \Omega^{2} S^{3}$. Let $\eta: L \rightarrow B O$ be the unique (up to homotopy) double loop map that induces an isomorphism on $H_{1}\left({ }_{-} ; \mathbb{Z} / 2\right)$.

(2.8) Lemma [19]. The Thom spectrum $T(\eta)$ is equivalent to the EilenbergMacLane spectrum $H_{0}$.

Now let $X=B \times L$ and let $\gamma$ be the stable vector bundle $\xi \times \eta$. Note that $v_{n+1}(\gamma) \neq 0$ so there is no Kervaire invariant in $\gamma$-cobordism theorem. Following the idea of Browder [2] we get around this as follows. Let $X\left\langle v_{n+1}\right\rangle$ be the homotopy fiber of the map

$$
v_{n+1}(\gamma): X \rightarrow K_{n+1}
$$

determined by $(n+1)$ st Wu class, and let $\gamma\left\langle v_{n+1}\right\rangle$ be the pull back of $\gamma$ over $X\left\langle v_{n+1}\right\rangle$. Notice that the map

$$
f=1 \times v_{n+1}: X \rightarrow X \times K_{n+1}
$$

determines, up to homotopy, a map of pairs

$$
\left(X, X\left\langle v_{n+1}\right\rangle\right) \rightarrow\left(X \times K_{n+1}, X \times *\right)
$$

where $* \in K_{n+1}$ is the basepoint. By letting $T(\gamma, \gamma\langle n+1\rangle)$ denote the Thom spectrum quotient $T(\gamma) / T \gamma\langle n+1\rangle$ we get an induced map

$$
F: T\left(\gamma, \gamma\left\langle v_{n+1}\right\rangle\right) \rightarrow T\left(X \times K_{n+1}\right) / T X=T \gamma \wedge K_{n+1} .
$$

Let $A$ be the cohomology class $\chi\left(S q^{n+1}\right) u \otimes i_{n+1}+u \otimes S q^{n+1} i_{n+1}$ in $H^{n+1}\left(T \gamma \wedge K_{n+1}\right)$. It is easy to check that $F^{*} A=0$ and we therefore get a commutative diagram

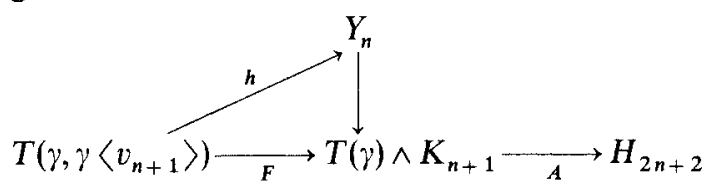

where $Y_{n}$ is the homotopy fiber of $A$. Let $\delta: T\left(\gamma, \gamma\left\langle v_{n+1}\right\rangle\right) \rightarrow \Sigma T\left(\gamma\left\langle v_{n+1}\right\rangle\right)$ be the connecting map for the cofibration sequence

$$
T\left(\gamma\left\langle v_{n+1}\right\rangle\right) \rightarrow T \gamma \rightarrow T\left(\gamma, \gamma\left\langle v_{n+1}\right\rangle\right)
$$

The following was a key technical lemma from Browder's work [2]. 
(2.9) Lemma [2]. a) $h_{*}: \pi_{j}^{s}\left(T\left(\gamma, \gamma\left\langle v_{n+1}\right\rangle\right)\right) \rightarrow \pi_{j}^{s} Y_{n}$ is an isomorphism for $j \leqq 2 n$ +1 .

b) If $x \in \pi_{2 n}^{s} T\left(\gamma\left\langle v_{n+1}\right\rangle\right)$, and $A F(x) \geqq 2$, then $x$ has nonzero Kervaire invariant if and only if there is an element $y \in \pi_{2 n+1}^{s}\left(T\left(\gamma, \gamma\left\langle v_{n+1}\right\rangle\right)\right.$ with $\delta_{*}(y)=x$ and such that $h_{*}(y)$ is the nontrivial class in the kernel of $i_{*}$ : $\pi_{2 k+1}^{s}\left(Y_{n}\right) \rightarrow \pi_{2 n+1}^{s} T(\gamma) \wedge K_{n+1}$.

Using Lemma (2.5) we can rewrite the $k$-invariant of $Y_{n}$.

(2.10) $A=\chi\left(S q^{n+1}\right) u \otimes i_{n+1}+u \otimes S q^{n+1} i_{n+1}=\sum_{i=0}^{n} S q^{n+1-i}\left(\psi_{\gamma}\left(v_{i}(\gamma)\right) \otimes i_{n+1}\right)$.

Now the inclusion $B \rightarrow L \times B=X$ yields a map of pairs ( $L$ $\times B, B) \rightarrow\left(X, X\left\langle v_{n+1}\right\rangle\right)$ because $v_{n+1}(\xi)=0$. Therefore by passing to Thom spectra and using the fact that $\gamma=\eta \times \xi$ and that $T \eta=H_{0}$ we get a map of relative Thom spectra

$$
R: T(\gamma, \xi)=\tilde{H}_{0} \wedge T(\xi) \rightarrow T\left(\gamma, \gamma\left\langle v_{n+1}\right\rangle\right) .
$$

Now given $\left.\alpha \in \pi_{2 n}^{s}(T)(\xi)\right)$ with $A(F) \geqq 2$ then there exists an element $\beta \in \pi_{2 n+1}^{s}\left(\tilde{H}_{0} \wedge T(\xi)\right)$ such that $\partial_{*} \beta=\alpha$. Then by combining (2.9) and (2.10) we see that $\alpha$ has nonzero Kervaire invariant if and only if the functional operation

$$
\left(\sum_{i=0}^{n} S q^{n+1-i}\right)_{\theta}\left(\psi_{\gamma}\left(v_{i}(\gamma)\right) \otimes i_{n+1}\right) \neq 0
$$

where $\theta=F_{*} R_{*}(\beta)$.

Recall that $\gamma=\eta \times \xi$. We will need the following lemma whose proof we postpone.

(2.12) Lemma. $v_{t}(\eta) v_{q}(\eta)=\left(\begin{array}{c}t+q \\ q\end{array}\right) v_{t+q}(\eta)$.

The proof of Theorem (2.4a) comes from the fact that

$$
0=R^{*} F^{*} A=\sum_{i=0}^{n} S q^{n+1-i} R^{*} F^{*}\left(\psi_{\gamma}\left(v_{i}(\gamma)\right) \otimes i_{n+1}\right)
$$

and thus, by (2.12) we have that

$$
\begin{aligned}
0 & =\sum_{i=0}^{n} S q^{n+1-i} R^{*} F^{*}\left(\psi_{\gamma}\left(v_{i}(\gamma)\right) \otimes i_{n+1}\right) \\
& =\sum_{i=0}^{n} S q^{n+1-i}\left(\psi_{(\gamma, \xi)}\left(v_{i}(\gamma) v_{n+1}(\gamma)\right)\right. \\
& =\sum_{i=0}^{n} S q^{n+1-i}\left(\psi_{(\gamma, \xi)} \sum_{\substack{s+t=i \\
p+q=n+1}}\left(\begin{array}{c}
t+q \\
q
\end{array}\right) v_{t+q}(\eta) \otimes v_{s}(\xi) v_{p}(\xi)\right) \\
& =\sum_{i=0}^{n} S q^{n+1-i}\left(\sum_{\substack{s+t=i \\
p+q=n+1}}\left(\begin{array}{c}
t+q \\
q
\end{array}\right) \chi\left(S q^{t+q}\right) \otimes \psi_{\xi}\left(v_{s}(\xi) v_{p}(\xi)\right)\right) .
\end{aligned}
$$


In the above calculation the notation $\psi_{(\gamma, \xi)}$ stands for the relative Thom isomorphism

$$
\psi_{(\gamma, \xi)}: H^{*}(X, B) \rightarrow H^{*}(T(\gamma, \xi)) .
$$

The proof of Theorem $(2.4 \mathrm{~b})$ uses this same calculation. Namely, by (2.11) and the naturality of functional cohomology operations, $\alpha \in \pi_{2 n}^{s}(T(\xi))$ with $A F(\alpha) \geqq 2$ has nonzero Kervaire invariant if and only if there is a class $\beta \in \pi_{2 n+1}^{s}\left(\tilde{H}_{0} \wedge T(\xi)\right)$ with

$$
0 \neq\left(\sum_{i=0}^{n} S q^{n+1-i}\right)_{\beta}\left(R^{*} F^{*} \psi_{\alpha}\left(v_{i}(\gamma)\right) \otimes i_{n+1}\right),
$$

which, by the above calculation occurs if and only if

$$
0 \neq\left(\sum_{i=0}^{n} S q^{n+1-i}\right)_{\beta}\left(\sum_{\substack{s+t=i \\
p+q=n+1}}\left(\begin{array}{c}
t+q \\
q
\end{array}\right) \chi\left(S q^{t+q}\right) \otimes \psi_{\xi}\left(v_{s}(\xi) v_{p}(\xi)\right)\right) .
$$

We are therefore reduced to proving Lemma (2.12). To do this, consider the Milnor basis, $\mathbb{Z} / 2\left[\zeta_{1}, \zeta_{2}, \ldots\right]$, for the dual of the Steenrod algebra, $A_{*}=H_{*} H$ $=H_{*} T(\eta)$. Recall that the evaluation $\left\langle S q^{i}, \zeta^{I}\right\rangle$ is nonzero if and only if $\zeta^{I}=\zeta_{1}^{i}$. Thus if we define the class $t_{i}=\chi\left(\zeta_{i}\right) \in H_{*} H=H_{*}(T(\eta))$, we have that the evaluation $\left\langle\chi\left(S q^{i}\right), t^{I}\right\rangle$ is nonzero if and only if $t^{I}=t_{1}^{i}$. Now let $x_{i} \in H_{*}\left(\Omega^{2} S^{3}\right)$ be the Thom isomorphic image of $t_{i} \in H_{*}(T(\gamma))$. Thus $H_{*}\left(\Omega^{2} S^{3}\right)=\mathbb{Z}_{2}\left[x_{1}, x_{2}, \ldots\right]$, and since $\gamma: \Omega^{2} S^{3} \rightarrow B O$ is an $H$-map, the multiplication in this algebra is induced by the loop addition in $\Omega^{2} S^{3}$. Moreover we have that the evaluation

$$
\left\langle v_{i}(\eta), x^{I}\right\rangle=1
$$

if and only if $x^{I}=x_{1}^{i}$. To prove (2.12) it is therefore sufficient to prove that

$$
\left\langle v_{t}(\eta) v_{q}(\eta), x^{I}\right\rangle=\left\{\begin{array}{cl}
\left(\begin{array}{c}
t+q \\
q
\end{array}\right) & \text { if } x^{I}=x_{1}^{t+q} \\
0 & \text { if } x^{I} \text { is any other monomial. }
\end{array}\right.
$$

To check this, let $\Delta: \Omega^{2} S^{3} \rightarrow \Omega^{2} S^{3} \times \Omega^{2} S^{3}$ be the diagonal map. Then

$$
\begin{aligned}
\left\langle v_{t}(\eta) v_{q}(\eta), x^{I}\right\rangle & =\left\langle\Delta^{*}\left(v_{t}(\eta) \otimes v_{q}(\eta)\right), x^{I}\right\rangle \\
& =\left\langle v_{t}(\eta) \otimes v_{q}(\eta) ; \Delta_{*} x^{I}\right\rangle
\end{aligned}
$$

which, by (2.13) is nonzero if and only if $\Delta_{*} x^{I}$ has as a summand $x_{1}^{t} \otimes x_{1}^{1}$. Lemma (2.12) now clearly follows.

\section{§3. Proofs of Theorems (1.6) and (1.8)}

In this section we evaluate the Kervaire invariant in the cobordism theories $\Omega_{*}^{O(1)}$ and $\Omega_{*}^{S O(2)}$ and prove Theorems (1.6) and (1.8). To do this we interpret the relations in (2.3) and (2.4) as elements in certain Tor groups, and the existence conditions in (2.3) and (2.4) as statements that certain elements in Ext groups 
are infinite cycles in the Adams spectral sequence. The most convenient vehicle for this step is the bar resolution, so we begin by recalling some basic facts about this resolution.

Let $M$ be an $A$ module and give $M \otimes A$ the diagonal $A$-action. Let $M \otimes A$ denote the free $A$-module which, as a $\mathbb{Z} / 2$-vector space is $M \otimes A$, and has $A$ action given by the formula $a(m \otimes b)=m \otimes a b$. There is a well known $A$ module isomorphism $F: M \otimes A \rightarrow M \otimes A$ induced by formula (2.5).

$$
F(m \otimes a)=\sum_{i} \chi\left(a_{i}^{\prime}\right)(m) \otimes a_{i}^{\prime \prime}
$$

where $a \rightarrow \sum_{i} a_{i}^{\prime} \otimes a_{i}^{\prime \prime}$ is the Cartan coproduct of the element $a \in A$.

Thus $M \otimes A$ is a free $A$-module and as is well known, the following chain complex, the reduced bar resolution, has as its homology the groups $\operatorname{Tor}_{A}^{i}\left(M ; \mathbb{Z}_{2}\right)$.

$$
M \overleftarrow{d_{1}} M \otimes \tilde{A} \longleftarrow_{d_{2}} M \otimes \tilde{A} \otimes \tilde{A} \longleftarrow \ldots \overleftarrow{d_{t}} M \otimes \tilde{A}^{i} \ldots
$$

where $\tilde{A}^{i}$ in the $i$-fold tenor product of the augmentation ideal $\tilde{A}$ with itself, and the differentials $d_{i}$ are given by the formula

$$
\begin{aligned}
d_{i}\left(m \otimes a_{1} \otimes \ldots \otimes a_{i}\right) & =a_{1}(m) \otimes a_{2} \otimes \ldots \otimes a_{i} \\
& +\sum_{j=1}^{i-1} m \otimes a_{1} \otimes \ldots \otimes a_{j-1} \otimes a_{j} a_{j+1} \otimes a_{j+2} \otimes \ldots \otimes a_{i} \in M \otimes \tilde{A}^{i-1} .
\end{aligned}
$$

Now let $M=H^{*}(T(\xi))$ as in Sect.2, then the relations in Theorem(2.3a) determine cycles $\mu_{2 n}(x) \in\left[H^{*}(T(\xi))\right] \otimes A$ as follows:

$$
\mu_{2 n}(x)=\sum_{i=0}^{n} \psi\left(x \cdot v_{i}\right) \otimes S q^{n+1-i} .
$$

Here $x \in H^{n}(B), \psi=\psi_{\xi}$, and $v_{i}=v_{i}(\xi)$. The relations in Theorem (2.4a) similarly determine cycles $\kappa_{2 n} \in H^{*} T(\xi) \otimes \tilde{A} \otimes \tilde{A}=\tilde{A} \otimes \tilde{A} \otimes H^{*} T(\xi)$ as follows:

$$
\kappa_{2 n}=\sum_{i=0}^{n} S q^{n+1-i} \otimes F\left(\sum_{\substack{s+t=i \\
p+q=n+1}}\left(\begin{array}{c}
t+q \\
q
\end{array}\right) \chi\left(S q^{t+q}\right) \otimes \psi\left(v_{s} v_{p}\right)\right)
$$

or, applying formula (3.1) for $F: M \otimes A \rightarrow M \otimes A$ we have

$$
\begin{gathered}
\kappa_{2 n}=\sum_{i=0}^{n} S q^{n+1-i} \otimes \sum_{\substack{s+t=i \\
p+q=n+1 \\
0 \leqq j \leqq t+q \\
\\
\in \tilde{A} \otimes \tilde{A} \otimes H^{*}(T \xi) .}}\left(\begin{array}{c}
t+q \\
q
\end{array}\right) \chi\left(S q^{t+q-j}\right) \otimes S q^{j} \psi\left(v_{s} v_{p}\right) \\
\text {. }
\end{gathered}
$$

These cycles determine classes $\mu_{2 n} \in \operatorname{Tor}_{A}^{1,2 n+1}\left(H^{*} T(\xi), \mathbb{Z}_{2}\right)$ and $\kappa_{2 n} \in \operatorname{Tor}_{A}^{2,2 n+2}\left(H^{*} T(\xi), \mathbb{Z}_{2}\right)$ respectively. The following is an immediate translation of Theorems (2.3) and (2.4) into Adams spectral sequence language. 
(3.4) Theorem. If $x \in H^{n}(B)$, then there is a $\xi$-oriented manifold $(M ; \Phi)$ with $\Phi_{*}[M]=0$ and $q\left(\Phi^{*}(x)\right) \neq 0$ if and only if $\alpha(M ; \Phi) \in \pi_{*}(T(\xi))$ is represented in the $\bmod 2$ Adams spectral sequence by a class $\zeta \in \operatorname{Ext}_{A}^{1,2 n+1}\left(H^{*} T(\zeta), \mathbb{Z}_{2}\right)$ such that the evaluation, $\left\langle\zeta, \mu_{2 n}(x)\right\rangle$ is nonzero.

(3.5) Theorem. Let $(M, \Phi)$ be a $\xi$-manifold with $A F(\alpha(M, \Phi)) \geqq 2$. Then the Kervaire invariant $K(M, \Phi)$ is nonzero if and only if $\alpha(M, \Phi) \in \pi_{*}(T(\xi))$ is represented in the $\bmod 2$ Adams spectral sequence by a class $\theta \in \mathrm{Ext}_{A}^{2,2 n+2}\left(H^{*} T(\xi), \mathbb{Z}_{2}\right)$ such that the evaluation $\left\langle\theta, \kappa_{2 n}\right\rangle$ is nonzero.

We now apply Theorems (3.4) and (3.5) to the cobordism theories $\Omega_{*}^{O(1)}$ and $\Omega_{*}^{S O(2)}$. We first pursue the case $B=B O(1)$ and prove Theorem (1.6). We begin by recalling the calculation of $\operatorname{Ext}_{\dot{A}}^{s, t}\left(H^{*}(M O(1)), \mathbb{Z}_{2}\right)=\operatorname{Ext}_{\boldsymbol{A}}^{s, t+1}\left(H^{*}\left(R P^{\infty}\right) ; \mathbb{Z}_{2}\right)$ from [12].

(3.6) Theorem. a) $\operatorname{Ext}_{A}^{0, t}\left(H^{*} M O(1): \mathbb{Z} / 2\right)=\left\{\begin{array}{l}\mathbb{Z} / 2 \quad \text { if } t=2^{i}-2 \\ \text { generated by a class } e_{2^{2}-2} \\ 0 \quad \text { if } t \neq 2^{i}-2 .\end{array}\right.$

b) $\operatorname{Ext}_{A}^{1, *}\left(H^{*} M O(1) ; \mathbb{Z} / 2\right)$ is the $\mathbb{Z} / 2$ vector space generated by class $e_{2^{i}-2} h_{j}$ represented by the classes $e_{2^{r}-2} \otimes \zeta_{1}^{2 i}$ in the cobar resolution, subject to the relations $e_{2^{i+1}-2} h_{i}=0$.

c) $\operatorname{Ext}_{A}^{2, *}\left(H^{*}(M O(1)) ; \mathbb{Z}_{2}\right)$ is the $\mathbb{Z} / 2$ vector space generated by classes $e_{2^{x}-2} h_{j} h_{k}$ and by classes $\bar{c}_{i}$ represented by

$$
\begin{aligned}
e_{3 \cdot 2-2} \otimes \zeta_{1}^{2^{1+2}} \otimes & \zeta_{1}^{2^{i+2}}+e_{2^{1+1}-2} \otimes\left(\zeta_{1}^{2^{1+2}} \otimes \zeta_{1}^{2^{2+1}} \zeta_{2}^{2^{2}}\right. \\
& \left.+\zeta_{2}^{2^{i+1}} \otimes\left(\zeta_{1}^{3 \cdot 2^{i}}+\zeta_{2}^{2^{t}}\right)+\zeta_{3}^{2^{1}} \otimes \zeta_{1}^{2^{2+1}}\right)
\end{aligned}
$$

in the cobar resolution. These classes are subject to the following relations:

(1) $e_{2^{i}-2} h_{i} h_{j}=e_{2^{i}-2} h_{j} h_{i}$

(2) $e_{2 r_{-1}} h_{i} h_{i+1}=0$ for all $r$

(3) $e_{2 r_{-1}} h_{1-1} h_{k}=0$ for all $k$

(4) $e_{2^{i+2}-1} h_{i}^{2}=e_{2^{i+1}-1} h_{i+1}^{2}$.

(5) $e_{2 i+2-1} h_{i+2} h_{i}=0$.

We remark that the cobar resolution is the dual of the bar resolution and the $\zeta_{i}$ 's are the Milnor ring generators of $A_{*}$ [22].

We now follow our procedure for detecting the Kervaire invariant.

Recall the Kahn-Priddy map $\lambda: R P^{\infty} \rightarrow S^{0}$. It is well known that on the Adams spectral sequence level $\lambda$ raises filtration by one, and induces the homomorphism $\operatorname{Ext}_{A}^{0, t}\left(H^{*} M O(1), \mathbb{Z} / 2\right)=\operatorname{Ext}_{A}^{0, t+1}\left(H^{*} R P^{\infty} ; \mathbb{Z} / 2\right) \rightarrow \operatorname{Ext}_{A}^{1, t+1}(\mathbb{Z} / 2, \mathbb{Z} / 2)$ given by $e_{2^{i}-2} \rightarrow h_{i}$. Thus by the Hopf invariant one results of [0], $e_{2^{2}-2}$ is an Adams spectral sequence permanent cycle if and only if $1 \leqq i \leqq 3$. Thus by Lemma (2.1), the Kervaire invariant $K_{h}(x) \equiv 0 \bmod 2$ for all $x \in \Omega_{2 n}^{O(1)}$ for $n>3$. Moreover, recall that $\left.v_{m} O(1)\right)$ is nonzero if and only if $m$ is of the form $2^{k}-1$. Thus $v_{1}^{2}(O(1))$ and $v_{3}^{2}(O(1))$ are nonzero and hence by (2.1)

$$
K_{h}: \Omega_{2 n}(\mathrm{O}(1)) \rightarrow \mathbb{Z} / 8
$$


is surjective for $n=1,3$ and is congruent to $0 \bmod 4$ for all other $n$. Using the fact that $\Omega_{2}^{O(1)}=\pi_{3}^{s}\left(\mathbb{R} P^{\infty}\right) \cong \mathbb{Z} / 8$ Theorem (1.6) parts b-d are proved.

By Theorem (2.4b) we are now reduced to computing the evaluations $\left\langle x ; \kappa_{2 n}\right\rangle$ for $x \in \operatorname{Ext}_{A}^{2,2 n+2}\left(H^{*} M O(1) ; \mathbb{Z}_{2}\right)$. Our first result is the following:

(3.7) Lemma. $\left\langle\bar{c}_{r} ; \kappa_{2 n}\right\rangle=0$ for all $r$.

Proof. We calculate this evaluation directly from the bar and cobar resolution descriptions of $\kappa_{2 n}$ and $\bar{c}_{,}$given in (3.3) and (3.6) respectively.

$\bar{c}_{r}$ has bigrading $\left(2,11 \cdot 2^{x}-2\right)$ so that we must have $2 n+2=11 \cdot 2^{\prime}-2$; i.e. $n$ $=11 \cdot 2^{r-1}-2$. Recall that

$$
\bar{c}_{1}=e_{3 \cdot 2^{r-2}} \otimes \zeta_{1}^{2 r+2} \otimes \zeta_{1}^{2 r+2}+e_{2^{r+1}-2} \otimes P_{1}
$$

where $P_{r} \in A^{*} \otimes A^{*}$ is given by

$$
P_{r}=\zeta_{1}^{2 r+2} \otimes \zeta_{1}^{2 r+1} \zeta_{2}^{2 r}+\zeta_{2}^{2 r+1} \otimes\left(\zeta_{1}^{3 \cdot 2 r}+\zeta_{2}^{2^{r}}\right)+\zeta_{3}^{2 r} \otimes \zeta_{1}^{2 r+1}
$$

We will prove this lemma in two steps, that is, we will show that both

$$
\left\langle e_{3 \cdot 2 r_{-2}} \otimes \zeta_{1}^{2 r+2} \otimes \zeta_{1}^{2 r+2} ; \kappa_{11 \cdot 2^{r-4}}\right\rangle \text { and }\left\langle e_{2^{r+1}-2} \otimes P_{r} ; \kappa_{11 \cdot 22_{-4}}\right\rangle
$$

are zero.

Now recall that

$$
\kappa_{2 n}=\sum_{\substack{0 \leq t \leq n \\
s \leq t=i \\
p+q=n+1 \\
0 \leqq j \leqq t+q}}\left(\begin{array}{c}
t+q \\
q
\end{array}\right) S q^{j} \psi\left(v_{s} v_{p}\right) \otimes \chi\left(S q^{t+q-j}\right) \otimes S q^{n+1-i}
$$

Thus after observing that $v_{k}(O(1))=0$ if and only if $k=2^{m}-1$ we note that in evaluating

$$
\left\langle e_{3 \cdot 2 r-2} \otimes \zeta_{1}^{2 r+2} \otimes \zeta_{1}^{2 r+2} ; \kappa_{11 \cdot 2 r_{-4}}\right\rangle
$$

we need only consider the summands of $K_{11 \cdot 2^{r}-4}$ where $\left\langle S q^{j} \psi\left(v_{s} v_{p}\right\rangle, e_{3 \cdot 2^{r-2}}\right\rangle$ $=1$ where $s$ and $p$ are both of the form $2^{m}-1$. This occurs in three cases

1. $j=2^{r}, s=p=2^{r}-1 ; 2 . j=0, s=2^{r+1}-1, p=2^{r}-1$;

3. $j=0, s=2^{r}-1, p=2^{r+1}-1$.

We study case 1 first. To get nonzero in the evaluation of this summand, we need that both

$$
\left\langle\zeta_{1}^{2 r+2} ;\left(\begin{array}{c}
t+q \\
q
\end{array}\right) \chi\left(S q^{t+q-2^{r}}\right)\right\rangle \text { and }\left\langle\zeta_{1}^{2^{r+2}} ; S q^{11 \cdot 2^{r-1}-1-i}\right\rangle
$$

are nonzero. In particular we need that $t+q-2^{r}=2^{r+2}$ and $11 \cdot 2^{r-1}-1-i$ $=2^{r+2}$. But using the restriction that $p+q=n+1$, i.e. that $2^{r}-1+q=11 \cdot 2^{r-1}$ $-1 ; q=9 \cdot 2^{r-1}$, we have that the binomial coefficient $\left(\begin{array}{c}t+q \\ q\end{array}\right)=\left(\begin{array}{c}2^{r+2}+2^{r} \\ 9 \cdot 2^{r-1}\end{array}\right) \equiv 0$. 
Thus the summands of $\kappa_{11 \cdot 2^{r-4}}$ when $j=2^{\prime}$, and $s=p=2^{\prime}-1$ evaluate trivially on $e_{3 \cdot 2^{r-2}} \otimes \zeta_{1}^{2^{r+2}} \otimes \zeta_{1}^{2 r+2}$. The fact that we get zero evaluations in the other two cases as well is verified in the same manner. This then shows that

$$
\left\langle e_{3 \cdot 2^{r}-2} \otimes \zeta_{1}^{2 r+2} \otimes \zeta_{1}^{2 r+2} ; \kappa_{11 \cdot 2^{r-4}}\right\rangle=0 .
$$

Now in evaluating $\left\langle e_{2^{r+1}-2} \otimes P_{1} ; \kappa_{11 \cdot 2^{r}-4}\right\rangle$ we need only consider the summands of $\kappa_{11 \cdot 2 r_{-4}}$ where $\left\langle S q^{j} \psi\left(v_{s} v_{p}\right) ; e_{2^{r+1-2}}\right\rangle=1$ where $s$ and $p$ are both of the form $2^{m}-1$. This occurs only when $j=0, s=p=2^{r}-1$. The fact that we get zero evaluations even on these summands is verified in a straightforward manner as above. In this case Milnor's result [22] that if $\zeta^{1}$ is a monomial in the $\zeta_{\text {,'s }}$ in $A^{*}$, then $\left\langle\zeta^{I} ; S q^{k}\right\rangle=1$ if and only if $\zeta^{I}=\xi_{1}^{k}$ is helpful. Details are left to the reader. This completes the proof of Lemma (3.7).

We now complete the calculation of $\left\langle x, \kappa_{2 n}\right\rangle$ for $x \in \mathrm{Ext}_{A}^{2,2 n+2}\left(H^{*} M O(1) ; \mathbb{Z} / 2\right)$ by proving the following:

(3.8) Lemma. $\left\langle e_{2^{r}-2} h_{k} h_{m} ; \kappa_{2 n}\right\rangle=1$ if and only if $k=m \neq r-1$, and $n=2^{m}$ $+2^{\prime-1}-2$.

Proof. In evaluating $\left\langle e_{2 r_{-2}} \otimes \zeta_{1}^{2^{\kappa}} \otimes \zeta_{1}^{2^{m}} ; \kappa_{2 n}\right\rangle$ we need only consider summands of $\kappa_{2 n}\left(n=2^{r-1}+2^{k-1}+2^{m-1}-2\right)$ where $\left\langle S q^{j} \psi\left(v_{s} v_{p}\right) ; e_{2^{r}-2}\right\rangle=1$ where $s$ and $p$ are both of the form $2^{u}-1$. This occurs only when $j=0$ and $s=p=2^{r-1}-1$. To get nonzero in the evaluation of this summand we need that both

$$
\left\langle\zeta_{1}^{2^{k}} ;\left(\begin{array}{c}
t+q \\
q
\end{array}\right) \chi\left(S q^{t+q}\right)\right\rangle \text { and }\left\langle\zeta_{1}^{2^{m}} ; S q^{2^{r-1}+2^{k-1}+2^{m-1}-1-i}\right\rangle
$$

are nonzero. In particular, we need that $t+q=2^{k}$ and $2^{i-1}+2^{k-1}+2^{m-1}-1-i$ $=2^{m}$. Now the binomial coefficient $\left(\begin{array}{c}t+q \\ q\end{array}\right)=\left(\begin{array}{c}2^{k} \\ q\end{array}\right)$ which is zero $(\bmod 2)$ unless $q=0$ or $2^{k}$. We are therefore reduced to two cases.

a) $j=0, s=p=2^{r-1}-1, q=0, t=2^{k}$, and

b) $j=0, s=p=2^{r-1}-1, q=2^{k}$, and $t=0$.

Now in case a), the two requirements that $2^{r-1}+2^{k-1}+2^{m-1}-1-i=2^{m}$ and $s$ $+t=i\left(2^{r-1}+2^{k}-1=i\right)$ yield a contradiction. We are thereby reduced to case b.

In this case the two requirements $2^{r-1}+2^{k-1}+2^{m-1}-1-i=2^{m}$ and $s+t=i$ $\left(2^{\prime-1}-1=i\right)$ yield that $k=m$. And indeed in this case both

$$
\left\langle\zeta_{1}^{2^{k}} ;\left(\begin{array}{c}
t+q \\
q
\end{array}\right) \chi\left(S q^{t+q}\right)\right\rangle=\left\langle\zeta_{1}^{2^{m}} ; S q^{2^{r-1}+2^{k-1}+2^{m-1}-i}\right\rangle=1
$$

so that $\left\langle e_{2^{r}-2} \otimes \zeta_{1}^{2^{k}} \otimes \zeta_{1}^{2^{m}} ; K_{2^{r}+2^{k+m}-4}\right\rangle=1$ if and only if $k=m$, which completes the proof of Lemma (3.8).

We've therefore completed our calculations of the evaluations $\left\langle x ; \kappa_{2 n}\right\rangle$ for $x \in \operatorname{Ext}_{A}^{2,2 n+2}\left(H^{*}(M O(1)) ; \mathbb{Z}_{2}\right)$. In fact we've shown that this evaluation is nonzero if and only if $x=e_{2^{m}-2} h_{k} h_{k}$ and $n=2^{k}+2^{m-1}-2$. Thus to complete the proof of Theorem (1.6) it suffices to prove the following. 
(3.9) Lemma. $e_{2 m_{-2}} h_{k} h_{k} \in \operatorname{Ext}_{A}^{2}\left(H^{*}(M O(1)): \mathbb{Z} / 2\right)$ can be an infinite cycle in the $\bmod 2$ Adams spectral sequence for $\pi_{*}(M O(1))$ only in the cases $m=k, m=k-1$, $m \leqq 3$ and $k$ arbitrary, or $k \leqq 2$ and $m$ arbitrary.

Proof. Consider the Kahn-Priddy map $\lambda_{*}: \operatorname{Ext}_{A}^{2, q}\left(H^{*} M O(1): \mathbb{Z}_{2}\right)=\operatorname{Ext}_{A}^{2, q+1}$ $\left(H^{*} R P^{\infty} ; \mathbb{Z}_{2}\right) \rightarrow \operatorname{Ext}_{A}^{3, q}\left(\mathbb{Z}_{2}, \mathbb{Z}_{2}\right)$. Then $\lambda_{*}\left(e_{2^{m_{-2}}} h_{k} h_{k}\right)=h_{m} h_{k} h_{k}$, so if $e_{2^{m_{-}}} h_{k} h_{k}$ is an infinite cycle, so it $h_{m} h_{k} h_{k}$ (in the Adams spectral sequence for $\pi_{*}^{s}\left(S^{0}\right)$.) Now as proved by Adams in [0] for $m \geqq 4$

$$
d_{2} h_{m}=h_{0} h_{m-1} h_{m-1} \text {. }
$$

Moreover, since $d_{2}\left(h_{k} h_{k}\right)=0$ we have that

$$
d_{2}\left(h_{m} h_{k} h_{k}\right)=h_{0} h_{m-1} h_{m-1} h_{k} h_{k}
$$

in $\operatorname{Ext}_{A}^{5, *}(\mathbb{Z} / 2, \mathbb{Z} / 2)$. Now except for the cases of $m$ and $k$ described in the statement of the lemma these classes were shown to be nonzero by W.H. Lin in [18]. Thus except for these cases $h_{m} h_{k} h_{k}$ carries a nonzero differential in the Adams spectral sequence, and hence so does $e_{2^{m}-2} h_{k} h_{k}$. This completes the proof of (3.9) and thus of Theorem (1.6).

The proof of Theorem(1.8) is completely analogous to the proof of (1.6) after observing that $\Sigma^{2} M S O(2) \cong \mathbb{C} P^{\infty}$. In this argument the calculation of $\operatorname{Ext}_{A}\left(H^{*} M S O(2) ; \mathbb{Z} / 2\right) \cong \operatorname{Ext}_{A}\left(H^{*}\left(\mathbb{C} P^{\infty}\right) ; \mathbb{Z} / 2\right)$ of [12] are used instead of (3.6). (For example, $\operatorname{Ext}_{A}^{2, *}\left(H^{*} M S O(2) ; \mathbb{Z} / 2\right)$ is generated by classes $e_{2^{m}-4} \otimes h_{k} h_{r}$ and $\bar{c}_{i}$ represented by

$$
\begin{gathered}
e_{3 \cdot 2^{i}-4} \otimes \zeta_{1}^{2^{i+2}} \otimes \zeta_{1}^{2^{i+2}}+e_{2^{1+1}-4} \otimes\left(\zeta_{1}^{2^{i+2}} \otimes \zeta_{1}^{2^{1+1}} \zeta_{2}^{2^{i}}+\zeta_{2}^{2^{i+1}} \otimes\left(\zeta_{1}^{3 \cdot 2^{2}}+\zeta_{2}^{2^{2}}\right)\right. \\
\left.+\zeta_{3}^{2^{i}} \otimes \zeta_{1}^{2^{i+1}}\right) .
\end{gathered}
$$

Taking the place of the Kahn-Priddy map we use the stable map $\lambda: \Sigma^{3} M S O(2)$ $=\mathbb{C} P^{\infty} \rightarrow S^{0}$ defined as follows.

Let $i_{n}: \mathbb{C} P^{n} \rightarrow \Omega U(n)$ be the usual inclusion map defined as follows. Fix a point $x \in \mathbb{C} P^{n}$. By considering $x$ as a complex line through the origin in $\mathbb{C}^{n}$, every vector $v \in \mathbb{C}^{n}$ can be written uniquely as $v=t+v^{\prime}$, where $t \in x$ and $t \cdot v^{\prime}=0$. Let $i_{n}(x) \in \Omega U(n)$ be defined by the requirement that if $z \in S^{1}$, then $i_{n}(x)(z)$ is the complex linear transformation which maps $v=t+v^{\prime}$ to $z t+v^{\prime}$.

Let $a\left(i_{n}\right): \Sigma \mathbb{C} P^{n} \rightarrow U(n)$ be the adjoint of $i_{n}$. These maps fit together to yield a map

$$
a(i): \Sigma \mathbb{C} P^{\infty} \rightarrow U
$$

Let $\lambda: \Sigma \mathbb{C} P^{\infty} \rightarrow S^{0}$ be the stable map defined to be the adjoint of the composition

$$
\lambda: \Sigma \mathbb{C} P^{\infty} \underset{a(i)}{\longrightarrow} U(n) \underset{J}{\longrightarrow} Q S^{0} .
$$

Here $Q S^{0}=\lim _{m \rightarrow \infty} \Omega^{m} \Sigma^{m} S^{0}$. A standard and easy calculation shows that, like the Kahn-Priddy map, $\lambda$ raises Adams filtration by one and the homomorphism

$$
\lambda_{*}: \operatorname{Ext}_{A}^{0}\left(H^{*} \operatorname{MSO}(2) ; \mathbb{Z} / 2\right) \rightarrow \operatorname{Ext}_{A}^{1}(\mathbb{Z} / 2, \mathbb{Z} / 2)
$$

is given by $e_{2^{m}-4} \rightarrow h_{m}$.

We leave the details of the proof of Theorem (1.8) to the interested reader. 


\section{§4. The Proof of Theorem(1.9) and some related results}

In order to construct elements $\theta_{j}(S O(2)) \in \Omega_{2^{j+1}-2}(S O(2)) \cong \pi_{2^{j+1}}^{s}\left(\mathbb{C} P^{\infty}\right)$ necessary to prove Theorem (1.9), we shall need to recall some techniques Mahowald used in his construction of elements $\eta_{j} \in \pi_{2 j}^{s}\left(S^{0}\right)$ represented by $h_{1} h_{j}$ $\in \operatorname{Ext}_{A}(\mathbb{Z} / 2, \mathbb{Z} / 2)$ in the Adams spectral sequence. We first fix some notation.

If $X$ is a space, let $Q X=\lim _{n \rightarrow \infty} \Omega^{n} \Sigma^{n} X$, and let $Q_{k} S^{0}$ denote the connected component of $Q S^{0}$ consisting of maps of degree $k$. Recall that $Q_{1} S^{0}$, a space often denoted by $F$ or $G$ in the literature, is an $H$-space, and in fact an infinite loop space, under the composition pairing of self maps of spheres.

Let $\eta: S^{1} \rightarrow Q_{1} S^{0}$ generate $\pi_{1} Q_{1} S^{0}=\pi_{1}^{s}\left(S^{0}\right)=\mathbb{Z} / 2$. Since $Q_{1} S^{0}$ is an infinite loop space $\eta$ extends to a two-fold loop map

$$
\tilde{\eta}: \Omega^{2} S^{3} \cong \Omega^{2} \Sigma^{2} S^{1} \rightarrow Q_{1} S^{0} .
$$

If $\Omega^{2} S_{+}^{3}$ denotes $\Omega^{2} S^{3}$ with a disjoint basepoint, then we have a basepoint preserving map

$$
\tilde{\eta}_{+}: \Omega^{2} S_{+}^{3} \rightarrow Q S^{0} .
$$

Let $g: \Omega^{2} S_{+}^{3} \rightarrow S^{0}$ be the stable map (i.e., map of the associated suspension spectra) given by the adjoint of $\tilde{\eta}_{+}$. By construction, $g$ is a map of ring spectra. In particular we may conclude the following.

Consider the stable splitting of $\Omega^{2} S_{+}^{3}$ due to Snaith [24]. (See [10] for a simple spectrum level proof.)

$$
\Omega^{2} S_{+}^{3} \simeq_{s} \underset{k \geqq 0}{\bigvee} D_{k}
$$

Here $D_{0}=S^{0}$, and for $k \geqq 1 D_{k}=F\left(\mathbb{R}^{2}, k\right)_{+} \wedge_{\Sigma_{k}} S^{1(k)}$, where $F\left(\mathbb{R}^{2}, k\right)$ is the configuration space of $k$-tuples of distinct points in $\mathbb{R}^{2}$. We then have commutative diagrams

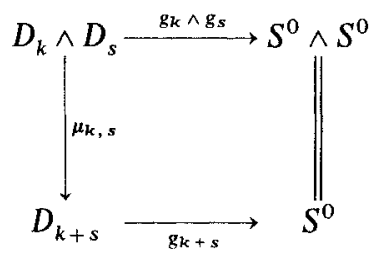

Here $g_{i}: D_{j} \rightarrow S^{0}$ is the restriction of $q$ to $D_{j}$, and the pairings $\mu_{k, s}$ are induced by the $H$-space structure of $\Omega^{2} S^{3}$.

The following two results were proven by Mahowald in [19].

(4.1) Lemma. $H^{*}\left(D_{2 k}\right)$ is isomorphic to the cyclic A-module

$$
M_{k}=A / A\left\{\chi\left(S q^{i}\right): i>k\right\}, \text { generated by a class } u_{2 k} \in H^{2 k}\left(D_{2 k}\right) .
$$

For ease of notation let $f_{i}=g_{2^{i}}: D_{2^{2}} \rightarrow S^{0}$. Consider the functional cohomology operation $S q_{f_{i}}^{2 i+1}$.

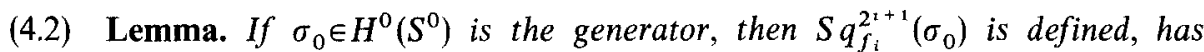
zero indeterminacy, and equals

$$
S q^{2^{i}-1}\left(u_{2^{i}}\right)=\chi\left(S q^{2^{i-1}} S q^{2^{i-2}} \ldots S q^{2^{1}} S q^{1}\right)\left(u_{2^{i}}\right) \in H^{*}\left(D_{2^{i}}\right) .
$$


We wish to reinterpret (4.2) as a statement about the representative of $f_{i}$ in the mod 2 Adams spectral sequence converging to the stable cohomotopy of $D_{2^{i}}$. To do this, first notice $H^{q}\left(D_{2^{i}}\right)=0$ for $q>2^{i+1}-1$, and $H^{2^{i+1}-1}\left(D_{2^{i}}\right)=\mathbb{Z} / 2$ generated by $S q^{2^{i}-1}\left(u_{2^{i}}\right)$. This implies that when localized at $2, D_{2^{i}}$ is $2^{i+1}-1$ dimensional, with only one cell in dimension $2^{i+1}-1$.

Let $p: D_{2^{i}} \rightarrow S^{2^{i+1}-1}$ be the projection onto the top cell. $p$ induces a homomorphism

$$
p_{*}: \operatorname{Ext}_{A}^{s, t}(\mathbb{Z} / 2, \mathbb{Z} / 2) \rightarrow \operatorname{Ext}_{A}^{s, t-2^{i+1}+1}\left(\mathbb{Z} / 2, H^{*}\left(D_{2^{i}}\right)\right) .
$$

(4.3) Corollary. $f_{i} \in \pi_{s}^{0}\left(D_{2^{i}}\right)$ is represented by $p_{*}\left(h_{i+1}\right) \in \operatorname{Ext}_{A}^{1,1}\left(\mathbb{Z} / 2, H^{*}\left(D_{2^{i}}\right)\right)$ in the mod 2 Adams spectral sequence converging to the stable cohomotopy of $D_{2}$, $\pi_{s}^{*}\left(D_{2^{i}}\right)$.

Proof. The translation of (4.2) to (4.3) is standard after noticing that for dimensional reasons (see (4.1)) if $j \neq i+1, S q_{f_{i}}^{2 j}\left(\sigma_{0}\right)=0$.

Now consider the stable map

$$
f_{i-1}^{(2)}: D_{2^{i-1}}^{(2)}=D_{2^{i-1}} \wedge D_{2^{i-1}} \underset{f_{i-1} \wedge f_{i-1}}{\longrightarrow} S^{0} \wedge S^{0}=S^{0} .
$$

The following is then immediate.

(4.4) Corollary. $f_{i-1}^{(2)} \in \pi_{s}^{0}\left(D_{2 i-1}^{(2)}\right)$ is represented by

$$
p_{*}^{(2)}\left(h_{i} h_{i}\right) \in \operatorname{Ext}_{A}^{2,2}\left(\mathbb{Z} / 2, H^{*}\left(D_{2^{i-1}}^{(2)}\right)\right),
$$

where

$$
p_{*}^{(2)}: \operatorname{Ext}_{A}^{s, t}(\mathbb{Z} / 2, \mathbb{Z} / 2) \rightarrow \operatorname{Ext}_{A}^{s, t-2^{i+1}+2}\left(\left(\mathbb{Z} / 2, H^{*}\left(D_{\left.2^{i}-1\right)}^{(2)}\right)\right)\right.
$$

is induced by the projection

$$
p^{(2)}: D_{2^{i-1}}^{(2)}=D_{2^{i-1}} \wedge D_{2^{i-1}} \stackrel{p \wedge p}{\longrightarrow} S^{2^{i}-1} \wedge S^{2^{i}-1}=S^{2^{i+1}-2} .
$$

Now let $\bar{D}_{2^{i}}$ be the $2^{i+1}-2$ skeleton of $D_{2 i}$. (All spaces and spectra are assumed to be localized at 2.) So

$$
\bar{D}_{2^{i}} \hookrightarrow D_{2^{i}} \stackrel{p}{\longrightarrow} S^{2^{i+1}-1}
$$

is a cofibration sequence. Notice that by (4.1), $H^{2^{i+1}-2}\left(\bar{D}_{2}\right)=\mathbb{Z} / 2$, generated by $S q^{2^{i}-2}\left(u_{2^{i}}\right)=\chi\left(S q^{2^{i-1}} S q^{2^{i-2}} \ldots S q^{2^{1}}\right)\left(u_{2^{i}}\right)$. Let $\bar{p}: \bar{D}_{2^{i}} \rightarrow S^{2 i+1}-2$ be the projection onto the top cell.

(4.5) Corollary. Let $\bar{f}_{i} \in \pi_{s}^{0}\left(\bar{D}_{2^{i}}\right)$ be the class represented by the restriction of $f_{i}$ to $\bar{D}_{2^{i}}$. Then $\bar{f}_{i}$ is represented by $\bar{p}_{*}\left(h_{i} h_{i}\right) \in \operatorname{Ext}_{A}^{2,2}\left(\left(\mathbb{Z} / 2, H^{*}\left(\bar{D}_{2^{i}}\right)\right)\right.$.

Proof. First notice that the complex $D_{2^{i-1}}^{(2)}$ is $2\left(2^{i}-1\right)=2^{i+1}-2$ dimensional, so we may assume the pairing $\mu=\mu_{2^{i-1}, 2^{i-1}}: D_{2^{i-1}}^{(2)} \rightarrow D_{2^{i}}$ has its image in $\bar{D}_{2^{i}}$. Furthermore, an easy exercise with 4.1 and the Cartan formula shows that the map

$$
\mu: D_{2^{i-1}}^{(2)} \rightarrow \bar{D}_{2^{i}}
$$


induces an injective map in cohomology. (This was verified in [7].) Thus the diagram

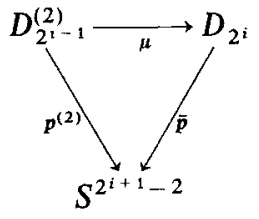

homotopy commutes (up to sign).

Now let

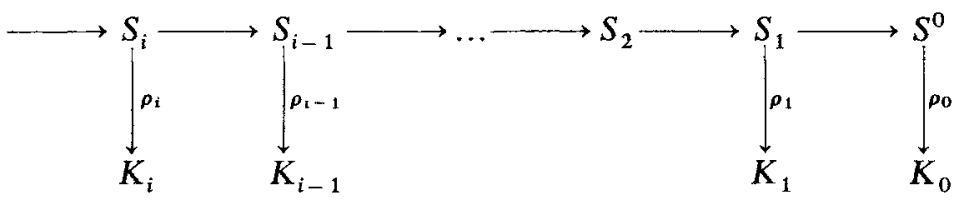

be a minimal Adams resolution of $S^{0}$. That is, it is an Adams resolution based on the minimal free resolution of $\mathbb{Z} / 2$ as a module over the Steenrod algebra. So the sequences $S_{i} \rightarrow S_{i-1} \rightarrow K_{i-1}$ are fibrations of spectra, with $K_{i}$ being a product of Eilenberg-MacLane spectra satisfying

$$
\pi_{q} K_{i} \cong \operatorname{Ext}_{\boldsymbol{A}}^{i, q+i}(\mathbb{Z} / 2, \mathbb{Z} / 2) .
$$

Now consider the following diagram.

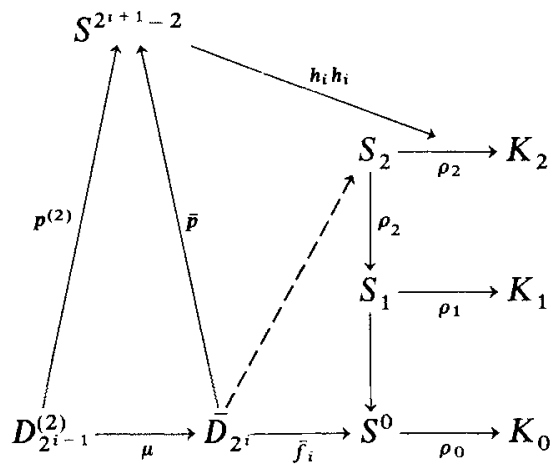

The map $\bar{f}_{i}: \bar{D}_{2 i} \rightarrow S^{0}$ lifts to a map $g_{i}: \bar{D}_{2^{i}} \rightarrow S_{2}$ since $\bar{f}_{i}$ is zero in cohomology, and since all functional operations $S q_{f_{i}}^{2^{k}}\left(\sigma_{0}\right)$ are zero. The map $h_{i} h_{i}$ : $S^{2^{i+1}-2} \rightarrow K_{2}$ represents the element of the same name in $\operatorname{Ext}_{A}^{2,2^{2+1}}(\mathbb{Z} / 2, \mathbb{Z} / 2)$.

Now by the commutativity of diagram (4.0), $\bar{f}_{i} \circ \mu=f_{i-1}^{(2)} \in \pi_{s}^{0}\left(D_{2^{i-1}}^{(2)}\right)$, and so by (4.4), $h_{i} h_{i} \circ p^{(2)}=\rho_{2} \circ g_{i} \circ \mu=\mu^{*}\left(\rho_{2} \circ g_{i}\right)$, where $\rho_{2} \circ g_{i} \in\left[\bar{D}_{2^{i}}, K_{2}\right]$. But $p^{(2)}=\bar{p} \circ \mu$ so we conclude that

$$
\mu^{*}\left(\rho_{2} \circ g_{i}\right)=\mu^{*}\left(h_{i} h_{i} \circ \bar{p}\right) .
$$

But since $\mu^{*}$ is injective, this implies that $\rho_{2} \circ q_{i}=h_{i} h_{i} \circ \bar{p}$ which, by definition determines the class

$$
\bar{p}_{*}\left(h_{i} h_{i}\right) \in \operatorname{Ext}_{A} \mathbb{Z} / 2, H^{*}\left(\bar{D}_{2^{\prime}}\right),
$$

which proves (4.5). 
The suspension spectrum $D_{2^{i}}$ is a finite spectrum so it has a SpanierWhitehead $2^{i+1}$-dual, $D_{2 i}^{*}$. That is, $\pi_{q}^{s} D_{2^{i}}^{*}=\pi_{2}^{2 i+1-q}\left(D_{2 i}\right)$, etc.

Let $\phi_{i} \in \pi_{2^{i+1}}^{s}\left(D_{2^{i}}^{*}\right)$ be the dual of $f_{i} \in \pi_{s}^{0}\left(D_{2^{i}}\right)$, and let $l \in \pi_{1}\left(D_{2^{i}}^{*}\right)$ be the dual of $p \in \pi_{s}^{2 i+1}-1\left(D_{2^{i}}\right)$. The map $\imath: S^{1} \rightarrow D_{2^{i}}^{*}$ induces a map

$$
\iota_{*}: \operatorname{Ext}_{\boldsymbol{A}}^{s, t}(\mathbb{Z} / 2, \mathbb{Z} / 2) \rightarrow \operatorname{Ext}_{A}^{s, t+1}\left(H^{*}\left(D_{2 i}^{*}\right), \mathbb{Z} / 2\right) .
$$

(4.6) Corollary. $\phi_{i} \in \pi_{2^{i+1}}\left(D_{2^{i}}^{*}\right)$ is represented by ${ }_{*}\left(h_{i+1}\right) \in \operatorname{Ext}_{A}^{1,2^{2+1}+1}\left(H^{*}\left(D_{2^{2}}^{*}\right)\right.$, $\mathbb{Z} / 2$ ) in the Adams spectral sequence.

Proof. This follows from (4.3) and $S$-duality.

Now let $\bar{D}_{2^{i}}^{*}$ be the stable cofibre of $i: S^{1} \rightarrow D_{21}^{*}$. Since $l$ is the dual of $p$ : $D_{2^{i}} \rightarrow S^{2^{i+1}-1}, \bar{D}_{2^{2}}^{*}$ is the $2^{i+1}$-dual of $\bar{D}_{2^{i}}$.

Let $j \in \pi_{2}^{s}\left(\bar{D}_{2}^{*}\right)$ be the dual of $\bar{p} \in \pi_{s}^{2^{2+1}-2}\left(\bar{D}_{2 i}\right)$, and let $\bar{\phi}_{i} \in \pi_{2^{i+1}}^{s}\left(\bar{D}_{2 i}^{*}\right)$ be the dual of $\bar{f}_{i} \in \pi_{s}^{0}\left(\bar{D}_{2^{i}}\right)$. (Note $\bar{\phi}_{i}$ is the composite $S^{2^{i+1}} \underset{\phi_{i}}{\longrightarrow} D_{2^{i}}^{*} \rightarrow \bar{D}_{2^{i}}^{*}$.)

(4.7) Corollary. $\bar{\phi}_{i} \in \pi_{2^{+1}}^{\mathrm{s}}\left(\bar{D}_{2^{2}}^{*}\right)$ is represented by $j_{*}\left(h_{i} h_{i}\right) \in \operatorname{Ext}_{A^{2,2+1-2}}^{2{ }^{1+1}}\left(H^{*}\left(\bar{D}_{2^{2}}^{*}\right)\right.$, $\mathbb{Z} / 2$ ) in the Adams spectral sequence, where $j_{*}$ is the homomorphism induced by $j$ : $S^{2} \rightarrow \bar{D}_{2 i}^{*}$.

Proof. This follows from (4.5) and $S$-duality.

The results described above are slight variations of the results obtained by Mahowald in [19]. Since the writing of that paper, however, more results about the stable homotopy type of the spaces $D_{k}$ (and thereby $\Omega^{2} S^{3}$ ) have been obtained by Brown and Peterson [7] and by Cohen [9, 11]. The essential outcome of these results is that when localized at any prime, $D_{k}$ has the homotopy type of an appropriate Brown-Gitler spectrum $[6,11]$. In particular at the prime 2 Brown and Peterson proved the following. (Again all spaces and spectra are assumed to be localized at 2.)

(4.8) Proposition [7]. $D_{k}$ satisfies the following properties:

(a) Let $u_{k}: D_{k} \rightarrow H_{k}$ be the generator of $H^{*}\left(D_{k}\right)$ as a module over the Steenrod algebra. Then if $X$ is any C.W complex, the induced homomorphism.

$$
\left(u_{k}\right)_{*}: \pi_{q+k}^{s}\left(D_{k} \wedge X\right) \rightarrow H_{q}(X)
$$

is surjective for $q \leqq \tilde{k}$, where

$$
\bar{k}= \begin{cases}k & \text { if } k \text { is odd } \\ k+1 & \text { if } k \text { is even. }\end{cases}
$$

(b) Let $M$ be a closed manifold of dimension $n \leqq k$, and let $T\left(v_{M}\right)$ be the Thom spectrum of the stable normal bundle $v_{M}$ of $M$. (Again the Thom class $u_{M} \in H_{*}\left(T\left(v_{M}\right)\right)$ lies in dimension zero.) Then there is a stable map

$$
\hat{u}_{M}: \Sigma^{k} T\left(v_{M}\right) \rightarrow D_{k}
$$

that makes the following diagram commute. 


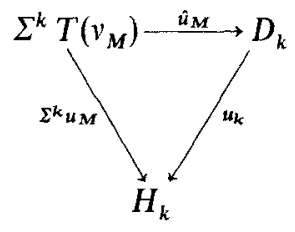

Remark. (b) follows from (a) by the $S$-duality relation between $M$ and $T\left(v_{M}\right)$.

(4.9) Corollary. Let $M$ be a closed $2^{i}$-dimensional manifold with Stiefel-Whitney class $\omega_{2^{2}-1}\left(v_{M}\right)$ nonzero in $H^{2^{2}-1}(M)$, then there is a stable map

$$
y_{M}: D_{2}^{*} \rightarrow M
$$

which is nonzero in $H_{1}\left({ }_{-}\right)$.

Proof. Let $y_{M}$ be the $S$-dual of the map $\tilde{u}_{M}: \Sigma^{2^{1}} T\left(v_{M}\right) \rightarrow D_{2^{i}}$ described in $(4.8 \mathrm{~b})$. Now $H_{1}\left(D_{21}^{*}\right) \cong H^{2^{i+1}-1}\left(D_{2}\right) \cong \mathbb{Z} / 2$ generated by $S q^{2^{i}-1}\left(u_{2^{2}}\right)$. So $y_{M}$ is nonzero on $H_{1}\left(_{-}\right)$if and only if $\tilde{u}_{M}^{*}\left(S q^{2^{i}-1}\left(u_{2}\right)\right)$ is nonzero.

But by $(4.8 \mathrm{~b})$,

$$
\begin{aligned}
\tilde{u}_{M}^{*}\left(S q^{2^{i}-1}\left(u_{2^{i}}\right)\right) & =S q^{2 i-1} \tilde{u}_{M}^{*}\left(u_{2^{i}}\right) \\
& =S q^{2^{i}-1}\left(u_{M}\right)
\end{aligned}
$$

which is nonzero since $\omega_{2^{i}-1}\left(v_{M}\right) \neq 0$.

(4.10) Corollary. For every $i>1$ there exists an element $\hat{\eta}_{1+1} \in \pi_{2^{i+1}}^{s}\left(R P^{2^{i}}\right)$ represented by $j_{*}\left(h_{i+1}\right) \in \mathrm{Ext}_{A}^{1,2^{i+1}+1}\left(H^{*} R P^{21}, \mathbb{Z} / 2\right) j_{*}$ is the homomorphism induced by the inclusion of the bottom cell $j: S^{1} \rightarrow \mathbb{R} P^{2 i}$.

Proof. Let $\hat{\eta}_{i+1}$ be the stable composition $s^{2^{i+1}} \underset{\phi_{1}}{\longrightarrow} D_{2^{i}}^{*} \underset{y_{i}}{\longrightarrow} R P^{2^{\imath}}$ where $\phi_{i}$ is as in 2.6 and $y_{i}=y_{R P^{21}}$ is as in (4.9). (Recall $\omega_{2^{2}-1}\left(v_{R P^{2}}\right) \neq 0$.)

The corollary follows from (4.6) and the fact that by (4.9) the following diagram homotopy commutes.

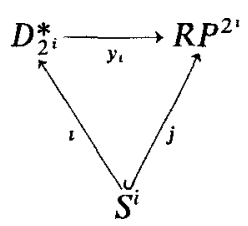

$\hat{\eta}_{i+1} \in \pi_{*}^{s}\left(R P^{2 x}\right)$ is nontrivial since $j_{*}\left(h_{i+1}\right) \in \operatorname{Ext}_{A}^{1}\left(H^{*} R P^{\infty}, \mathbb{Z} / 2\right)$ is nonzero, and cannot be hit by a differential in the Adams spectral sequence.

An interesting outcome of the above analysis is that we can recover Mahowald's theorem that $h_{1} h_{j} \in \operatorname{Ext}_{A}(\mathbb{Z} / 2, \mathbb{Z} / 2)$ is a permanent cycle in the Adams spectral sequence. That is, if we let $\eta_{j} \in \pi_{2^{j}}^{s}\left(S^{0}\right)$ be the composition

$$
\eta_{j}: S^{2^{j}} \underset{\hat{\eta}_{j}}{\longrightarrow} R P^{2^{j-1}} \subset R P^{\infty} \underset{\lambda}{\longrightarrow} S^{\Theta}
$$

where $\lambda$ is the map studied in the Kahn-Priddy theorem [16], then we have the following: 
(4.11) Corollary. $\eta_{j} \in \pi_{2^{j}}^{s}\left(S^{0}\right)$ is represented by $h_{1} h_{j} \in \mathrm{Ext}_{\boldsymbol{A}}^{2,2^{j}+2}(\mathbb{Z} / 2, \mathbb{Z} / 2)$ in the Adams spectral sequence.

Proof. This follows from (4.10) and the well known fact that $\lambda$, when restricted so $S^{1} \subset R P^{\infty}$, is the generator of $\pi_{1}^{s}\left(S^{0}\right)$, which is represented by $h_{1}$.

We now have all the necessary preliminary results to complete the proof of Theorem 2.

Let $\theta_{i}(S O(2)) \in \pi_{2^{i+1}}^{s}\left(\mathbb{C} P^{\infty}\right)=\pi_{2^{i+1}-2}^{s}(M S O(2))=\Omega_{2^{i+1-2}}(S O(2))$ be given by the following stable composition.

$$
\theta_{i}(S O(2)): S^{2^{i+1}} \underset{\hat{\eta}_{i+1}}{\longrightarrow} R P^{\infty} \stackrel{\rho}{\longrightarrow} R P_{2}^{\infty} \stackrel{\omega}{\longrightarrow} \mathbb{C} P^{\infty},
$$

where $R P_{2}^{\infty}$ is the mapping cone of the inclusion $j: S^{1} \subset R P^{\infty}$ and $\rho$ is the projection, and $\omega: R P_{2}^{\infty} \rightarrow \mathbb{C} P^{\infty}$ generates $H^{2}\left(R P_{2}^{\infty} ; \mathbb{Z}\right) \cong \mathbb{Z}$. We need to show that $\theta_{i}(S O(2))$ is represented by $u_{*}\left(h_{i} h_{i}\right) \in \mathrm{Ext}^{2,2^{i+1}+2}\left(\mathrm{CP}^{\infty}\right)$, where $u: S^{2} \rightarrow C P^{\infty}$ is the inclusion of the bottom cell.

Now since the map $y_{i}: \bar{D}_{2^{i}}^{*} \rightarrow R P^{\infty}$ induces an isomorphism on $H_{1}(\ldots)$ and hence on $\pi_{1}\left({ }_{-}\right)$, it induces a map

$$
\bar{y}_{i}: \bar{D}_{2^{i}}^{*} \rightarrow R P_{2}^{\infty},
$$

which we claim is an isomorphism on $H_{2}\left({ }_{-}\right)$. This is true since $H_{2}\left(\bar{D}_{2^{i}}^{*}\right)$ $\cong H^{2^{i+1}-2}\left(\bar{D}_{2^{i}}\right) \cong \mathbb{Z} / 2$ generated by $S q^{2^{i-2}}\left(u_{2^{i}}\right)$. So by $S$-duality $\bar{y}_{i}$ induces an isomorphism on $H_{2}\left({ }_{-}\right)$iff the Stiefel-Whitney class $\omega_{2^{i}-2}\left(v_{R P^{2}}\right)$ is nonzero. But this is standard.

Now since $\omega_{*}: H_{2}\left(R P_{2}^{\infty}\right) \rightarrow H_{2}\left(\mathbb{C} P^{\infty}\right)$ is an isomorphism, we may conclude that the following diagram homotopy commutes

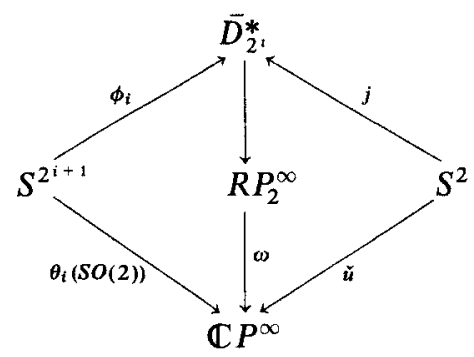

where $j$ and $\phi_{i}$ are in the Corollary (4.7). Theorem (1.9) now follows from the commutativity of this diagram and (4.7).

We conclude this section with a proof of the following theorem, originally observed by R. Bruner [8].

(4.12) Theorem. For every $j \geqq 5 h_{2} h_{j}^{2} \in \operatorname{Ext}_{A}^{3}(\mathbb{Z} / 2, \mathbb{Z} / 2)$ is an infinite cycle in the Adams spectral sequence.

Proof. Let $\lambda: \Sigma \mathbb{C} P^{\infty} \rightarrow S^{0}$ be the stable map described at the end of Sect. 2 . Define $\zeta_{j}$ to be the composition

$$
\zeta_{j}: S^{2 j+1}+\underset{\theta_{j}(S O(2))}{\longrightarrow} \Sigma^{3} M S O(2)=\Sigma \mathbb{C} P^{\infty} \underset{\lambda}{\longrightarrow} S^{0} .
$$


The fact that $\zeta_{j}$ is detected by $h_{2} h_{j} h_{j}$ is immediate from the fact that $\theta_{j}(S O(2))$ is detected by $u_{*}\left(h_{j} h_{j}\right)=e_{0} \otimes h_{j} h_{j} \in \operatorname{Ext}_{A}^{2}\left(H^{*} M S O(2) ; \mathbb{Z} / 2\right)$ and from the fact that, as observed at the end of Sect. $2, \lambda_{*}\left(e_{0}\right)=h_{2} \in \operatorname{Ext}_{A}^{1}(\mathbb{Z} / 2, \mathbb{Z} / 2)$.

\section{§5. Appendix: Algebra of quadratic forms}

In this appendix we do the Witt group calculations described in Sect.1. The set up for these calculations is the following.

$V$ is a $\mathbb{Z} / 2$ vector space.

$(x \cdot y)$ denotes the value of a nonsingular, symmetric pairing $V \times V \rightarrow \mathbb{Z} / 2$,

$A$ is an abelian group equipped with an embedding $i: \mathbb{Z} / 2 \rightarrow A$, and

$q: V \rightarrow A$ is a quadratic form; that is it satisfies $q(x+y)=q(x)+q(y)+i(x \cdot y)$

Notice that $2 q(x)=i(x \cdot x)$ and that $4 q(x)=0$. We therefore assume without loss of generality that $2 A \subset i(\mathbb{Z} / 2)$ and $4 A=0$. We write $A_{2} \subset A$ for the subgroup of elements of order $\leqq 2$.

Recall that $(V, q)$ was defined to be Witt equivalent to zero if there is a subspace $K \subset V$ such that 2 rank $K=\operatorname{rank} V$ and $q(K)=0 .\left(V_{1}, q_{1}\right)$ is Witt equivalent $\left(V_{2}, q_{2}\right)$ if $\left(V_{1} \oplus V_{2}, q_{1}-q_{2}\right)$ is Witt equivalent to zero.

N.B. i) If $\operatorname{rank}(V)$ is odd $V$ is never Witt equivalent to zero.

ii) It is easy to check that if $K \subset V$ and $q(K)=0$, then 2 rank $K \leqq$ rank $V$.

Our aim is to compute the Witt group, $W(A)$; the set of equivalence classes $[V, q]$ under addition given by $\left[V_{1}, q_{1}\right]+\left[V_{2}, q_{2}\right]=\left[V_{1} \oplus V_{2}, q_{1}+q_{2}\right]$.

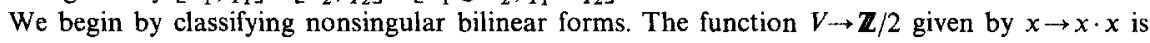
a homomorphism, hence by the nonsingularity of the pairing there exists a unique $v \in V$ such that $x \cdot x=x \cdot v$ for all $x \in V$. We call $v$ the Wu-class of $V$. We then divide nonsingular bilinear forms on $V$ into the following three types:

$$
\begin{array}{llll}
\text { I. } v=0 & \text { II. } v \neq 0, v \cdot v=0 & \text { III. } v \neq 0, v \cdot v=1
\end{array}
$$

We will classify these bilinear forms as direct sums of the following three basic ones. $v=0$.

1. Let $E=\mathbb{Z} / 2 \oplus \mathbb{Z} / 2$ with basis $x, y$ and bilinear form given by $x \cdot x=y \cdot y=0, x \cdot y=1$. Here

2. Let $F=\mathbb{Z} / 2 \oplus \mathbb{Z} / 2$ with basis $x, y$ and bilinear form $x \cdot x=0, x \cdot y=y \cdot y=1$. Here $v=x$.

3. Let $G=\mathbb{Z} / 2$ with basis $x$ and bilinear form $x \cdot x=1$. Here $v=x$.

(5.1) Lemma. Let $V$ be a vector space with nonsingular bilinear form $x \cdot y$.

a) $V$ is of type $I$ iff $V \cong \bigoplus_{i=1}^{n} E_{i}$.

b) $V$ is of type $I I$ iff $V \cong \bigoplus_{i=1}^{n} E_{i} \oplus F$.

c) $V$ is of type $I I I$ iff $V \cong \bigoplus_{i=1}^{n} E_{i} \oplus G$.

In all cases, isomorphism means bilinear form preserving isomorphism.

Before we prove this lemma we adopt the following notation.

1. If $x_{1}, \ldots, x_{k} \in V$ let $\left\langle x_{1}, \ldots, x_{k}\right\rangle$ denote the span of $x_{1}, \ldots, x_{k}$ in $V$.

2. If $W \subset V$ is a subspace, let $W^{\perp}=\{x \in V: x \cdot w=0$ for all $w \in W\}$. Note that in general $W \cap W^{\perp}$ $\neq 0$.

Proof. a) If $x \in V$ then by nonsingularity there is an element $y \in V$ such that $x \cdot y=1$. Since $V$ is of type $I$ this means $x$ and $y$ are linearly independent. Thus $V \cong\langle x, y\rangle \oplus\langle x, y\rangle^{\perp}$ and an obvious induction completes the proof.

b) Choose a $w$ such that $v \cdot w=1$. By assumption $v$ and $w$ are independent. Clearly $V=\langle v, w\rangle$ $\oplus\langle v, w\rangle^{\perp}$. If $x \in\langle v, w\rangle^{\perp}$, then $x \cdot x=v \cdot x=0$ so that $\langle v, w\rangle^{\perp}$ is of type I. By assumption $v \cdot v=0$ and $v \cdot w=1$. Now by the definition of $v, w \cdot w=v \cdot w=1$ so $\langle v, w\rangle \cong F$ and (b) now follows from (a).

c) Since $v \cdot v=1$, then clearly $V \cong\langle v\rangle \oplus\langle v\rangle^{\perp}$. Moreover one can check that $\langle v\rangle^{\perp}$ is of type $\mathrm{I}$ and it is obvious that $\langle v\rangle=G$. Part (1) therefore completes the proof.

This completes the classification of nonsingular bilinear forms. 
(5.2) Lemma. Let $v \in V$ be the $W u$ class and let $q: V \rightarrow A$ be a quadratic form. Then $q(v)=0$ iff $(V, q)$ is Witt equivalent to an $A_{2}$-valued quadratic form.

Proof. There are three cases according to the type of the bilinear form.

Case 1. $V$ is of type I: Since $v=0, x \cdot x=0$ for all $x \in V$. So $0=x \cdot x=2 q(x)$ and thus $q$ is $A_{2}$ valued.

Case 2. $V$ is of type II: Pick $w$ such that $v \cdot w=1$ and set $F=\langle v, w\rangle, P=\langle v, w\rangle^{\perp}, q_{F}=\left.q\right|_{F}$, and $q_{P}$ $=\left.q\right|_{P}$. Then $V=\left(F, q_{F}\right) \oplus\left(P, q_{P}\right)$. If we assume $q(v)=0$ then $\left(F, q_{F}\right) \sim 0$ so $(V, q) \sim\left(P, q_{P}\right)$. But $P$ is of type I, so as in case $1, q_{P}$ is $A_{2}$ valued.

We now assume that $q(v) \neq 0$ and will show that $q$ is not equivalent to an $A_{2}$ valued form. We compute $q_{F}$ :

$q_{F}(v) \neq 0$, by assumption. $q_{F}(v+w)=q_{F}(v)+q_{F}(w)+i(v \cdot w)$. Now $2 q_{F}(v)=0$ since $v \cdot v=0$. Moreover $2 i(v \cdot w)=0$, but $2 q_{F}(w) \neq 0$ since $w \cdot w=v \cdot w=1$. Therefore

$$
0 \neq 2 q_{F}(w)=2 q_{F}(v+w) .
$$

Now suppose $\left(Q, q_{Q}\right)$ is an $A_{2}$-valued form with $(V, q) \oplus\left(Q, q_{Q}\right) \sim 0$. Then there is a subspace $K \subset V \oplus Q$ with $2 \operatorname{rank} K=\operatorname{rank} V+\operatorname{rank} Q$ and such that $q+q_{Q}$ vanishes on $K$. But by the above calculation of $q_{F}$ we have that $K \cap F=0$ so that $K \subset P \oplus Q$. But by the remark at the beginning of this Sect. $2 \operatorname{rank} K \leqq \operatorname{rank} P+\operatorname{rank} Q<\operatorname{rank} V+\operatorname{rank} Q$. This contradiction shows that $(V, q)$ is not equivalent to an $A_{2}$-valued form.

Case 3. $V$ is of type III. By Lemma (5.1) we know that rank $V$ is odd. If $\left(Q, q_{Q}\right)$ is any $A_{2}$-valued form then $Q$ has type I since $0=2 q_{Q}(x)=i(x \cdot x)=x \cdot v$ for all $x$. Again by (5.1) this implies that $\left(Q, q_{Q}\right)$ has even rank. Therefore $V \oplus Q$ has odd rank and hence cannot be Witt equivalent to zero. Thus $(V, q)$ cannot be Witt equivalent to any such $\left(Q, q_{Q}\right)$.

Now define a homomorphism $\phi: W(A) \rightarrow A$ by $\Phi[V, q]=q(v)$. Lemma (5.2) implies that this is well defined since if $(V, q) \sim 0$ then $q(v)=0$. Clearly $\phi$ is a homomorphism.

Lemma. Suppose $2 A \neq 0$, then there is a short exact sequence

$$
0 \rightarrow W\left(A_{2}\right) \rightarrow W(A) \stackrel{\phi}{\longrightarrow} A \rightarrow 0
$$

This sequence is never split.

Proof. From (5.2) it is clear that this sequence is exact at $W(A)$. Moreover it is also clear that $W\left(A_{2}\right) \rightarrow W(A)$ is injective. We now show that $\phi$ is surjective.

Suppose first that $a \in A$ is such that $2 a \neq 0$. Define $i: \mathbb{Z} / 2 \rightarrow A$ by $i(1)=a$. Take the bilinear form $G$ and define $q(x)=a$. Clearly $\Phi[G, q]=a$.

Now assume $a \in A$ is such that $2 a=0$. Take the bilinear form $F$ and define $q$ by $q(x)=a, q(y)$ $=b$ where $b$ is an arbitrary element of $A$ of order 4 . (N.B. $y \cdot y \neq 0$ so $2 q(y) \neq 0$ ). Clearly $\phi[F, q]=a$. This shows that $\phi$ is onto.

To show that the sequence is not split we exhibit an element of $W(A)$ of order 8 .

Let $a \in A$ be an element of order 4. Define the form $(G, q)$ as above; namely, $q(x)=a$. Recall that $4(G, q)$ is the direct sum $(G, q) \oplus(G, q) \oplus(G, q) \oplus(G, q)$. Thus it has basis $x_{1}, x_{2}, x_{3}, x_{4}$ and the quadratic form $q_{4}=q \oplus q \oplus q \oplus q$ is given by the formulae:

$$
\begin{aligned}
q_{4}\left(x_{i}\right) & =a \text { for all } i, \quad q_{4}\left(x_{i}+x_{j}\right)=2 a \text { for } i \neq j, \\
q_{4}\left(x_{i}+x_{j}+x_{k}\right) & =3 a \text { for } i \neq j \neq k, \text { and } q_{4}\left(x_{1}+x_{2}+x_{3}+x_{4}\right)=0 .
\end{aligned}
$$

Hence $q_{4}$ vanishes on only two elements $\left(0\right.$ and $\left.x_{1}+x_{2}+x_{3}+x_{4}\right)$ and so it never vanishes on a rank two subspace. This shows that $4[G, q] \neq 0$, and completes the proof of Lemma (5.3).

Notice that (5.3) allows us to easily reduce the calculation of $W(A)$ to the calculation of $W\left(A_{2}\right)$. We therefore assume until further notice that $2 A=0$.

Let $h: A \rightarrow \mathbb{Z} / 2$ be any homomorphism such that $h i: \mathbb{Z} / 2 \rightarrow A \rightarrow \mathbb{Z} / 2$ is the identity. Then the function $q_{h}: V \rightarrow \mathbb{Z} / 2$ given by $h \circ q$ is quadratic and it therefore has an Arf invariant

$$
\operatorname{Arf}\left(q_{h}\right)=\operatorname{Arf}_{h}(q) \in \mathbf{Z} / 2
$$

Recall that Arf: $W(\mathbf{Z} / 2) \rightarrow \mathbf{Z} / 2$ is an isomorphism. This gives a homomorphism

$$
\text { Arf: } W(A) \rightarrow \underset{h}{\oplus} \mathbb{Z} / 2
$$

given by $(V, q) \rightarrow \Sigma \operatorname{Arf}_{h}(q)$. Here $h$ varies over those homomorphisms $h: V \rightarrow \mathbb{Z} / 2$ such that $h i=1$. 
(5.4) Lemma. Arf: $W(A) \rightarrow \underset{h}{\oplus} \mathbb{Z} / 2=(\mathbb{Z} / 2)^{2^{d-1}}$ is injective. Here $d=\operatorname{rank} A$.

Proof. We use induction on $d$. The case $d=1$ is the assertion that Arf: $W(\mathbb{Z} / 2) \rightarrow \mathbb{Z} / 2$ is an isomorphism.

Assume the result is true for $A$ and set $B=A \oplus \mathbb{Z} / 2$ where $i: \mathbb{Z} / 2 \rightarrow B$ is given by $\mathbb{Z} / 2 \rightarrow A \hookrightarrow B$. Let $q: V \rightarrow B$ be any quadratic form, then we can write

$$
q=q_{A} \oplus \psi
$$

where $q_{A}: V \rightarrow A$ is quadratic and $\psi: V \rightarrow \mathbb{Z} / 2$ is a homomorphism. Similarly, if $h: B \rightarrow \mathbb{Z} / 2$ is a homomorphism such that $h \circ i=1$, then $h=h_{A} \oplus \phi$, where $h_{A}: A \rightarrow \mathbb{Z} / 2$ is a homomorphism with $h_{A} i$ $=1$, and $\phi: \mathbb{Z} / 2 \rightarrow \mathbb{Z} / 2$ is any homomorphism. Then we have

$$
q_{h}(x)=q_{h_{A}}(x)+\phi \psi(x) .
$$

Now since $\phi \psi: V \rightarrow \mathbb{Z} / 2$ is linear, $\phi \psi(x)=x \cdot z$ for some unique $z \in V$, and

$$
q_{h}(x)=q_{h_{A}}(x)+x \cdot z .
$$

By a standard result on the Arf invariant

$$
\operatorname{Arf}\left(q_{k}\right)=\operatorname{Arf}\left(q_{h_{A}}\right)+q_{h}(z)
$$

(N.B. $d \cdot d=0$ since $2 A=0$, so that $q_{h_{A}}(z)=q_{h}(z)$ ).

We now show that Arf: $W(B) \rightarrow \underset{h}{\oplus} \mathbb{Z} / 2$ is injective. So suppose $\operatorname{Arf}\left(q_{h}\right)=0$ for all choices of $h$. By choosing $\phi=0$ so that $z=0$, we therefore have that $\operatorname{Arf} q_{h_{A}}=0$ for all suitable $h_{A}$, and so $\operatorname{Arf}\left(q_{A}\right)=0$. Thus by our inductive assumption; we have $\left[V, q_{A}\right]=0$ in $W(A)$.

This also shows that for all suitable choices of $h$

$$
0=\operatorname{Arf}\left(q_{h}\right)=q_{h}(d)
$$

By the definition of $d$ we have that $d$ must be zero and hence $\psi=0$. Therefore $q$ is the composite $V \underset{q_{A}}{\longrightarrow} A \hookrightarrow B$. However $\left[V, q_{A}\right]=0$ in $W(A)$ so there is a half rank subspace $K \subset V$ with $q_{A}(K)=0$. But then $q(K)=0$ so $[V, q]=0$ in $W(B)$. This completes the inductive step in our proof of (5.4).

We are now reduced to computing the rank of the image of Arf: $W(A) \rightarrow \oplus_{h} \mathbb{Z} / 2$.

We adopt the notation $\langle a, b\rangle$ for $a, b \in A$, for the quadratic form defined on the module $E$ by the formula $x \rightarrow a, y \rightarrow b$. It is clear that every quadratic form over $A$ (where $2 A=0$ ) is a direct sum of such forms. Write $[a, b] \in W(A)$ for the Witt class of $\langle a, b\rangle$. Thus $W(A)$ is generated by the elements $[a, b]$. There are certain obvious relations.

(5.5) Lemma. In $W(A)$, (i) $[a, b]=[b, a]$, (ii) $[0, b]=0$, (iii) $[a, b+c]=[a, b]+[a, c]$.

Proof. The only nontrivial part is (iii). To prove this we compute Arf invariants. Let $h: A \rightarrow \mathbb{Z} / 2$ be any homomorphism with $h i=1$. Then by definition,

$$
\begin{aligned}
\operatorname{Arf}_{h}\langle a, b+c\rangle & =q_{h}(x) q_{h}(y) \\
& =h(a) h(b+c) \\
& =h(a) h(b)+h(a) h(c) \\
& =\operatorname{Arf}_{h}\langle a, b\rangle+\operatorname{Arf}_{h}\langle a, c\rangle .
\end{aligned}
$$

Thus by $5.4[a, b+c]=[a, b]+[a, c]$.

We now set up some more notation. Pick a basis for $A,\left\{a_{1}, \ldots, a_{d}\right\}$ where $a_{1}=i(1)$. Let $\left\{h_{1}, \ldots, h_{d}\right\}$ be the dual basis for $A^{*}=\operatorname{Hom}(A ; \mathbb{Z} / 2)$. Let $H \subseteq A^{*}$ be the subspace consisting of those homomorphisms that vanish on $a_{1}$. So $H=\left\langle h_{2}, \ldots, h_{2}\right\rangle$. Thus if $h: A \rightarrow \mathbb{Z} / 2$ is such that $h i=1$, then $h \in h_{1}+H$.

Now from Lemma (5.5) we see that $W(A)$ is spanned by $\left[a_{i}, a_{j}\right]$ such that $1 \leqq i \leqq j \leqq d$. We need to extract a basis from this set.

(5.6) Lemma. $\left[a_{1}, a_{k}\right]=\left[a_{k}, a_{k}\right]$ for each $k$.

Proof. We compute Arf invariants. If $h \in h_{1}+H$, then $h\left(a_{1}\right)=1$, so $\operatorname{Arf}_{h}\left\langle a_{1}, a_{k}\right\rangle=h\left(a_{k}\right)=h\left(a_{k}\right) h\left(a_{k}\right)$ $=\operatorname{Arf}_{h}\left\langle a_{k}, a_{k}\right\rangle$. 
(5.7) Lemma. The set $\left\{\left[a_{1}, a_{1}\right]\right\} \cup\left\{\left[a_{i}, a_{j}\right]: 1 \leqq i<j \leqq d\right\}$ is a basis for $W(A)$.

Proof. By Lemmas (5.5) and (5.6) the above set spans $W(A)$. To show it is linearly independent order the elements $\left[a_{i}, a_{j}\right]$ in this set lexicographically. Now order the elements of $h_{1}+H$ as follows. Let $\phi, \psi \in h_{1}+H$. Define $l(\phi)$ to be the number of $h_{i}$ 's in the expression of $\phi$ as a linear combination of the $h_{i}$ 's. Then

(i) If $l(\phi)<l(\psi)$ then $\phi \leqq \psi$,

(ii) If $l(\phi)=l(\psi)$ then order $\phi$ and $\psi$ lexiocographically.

We introduce the following notation for elements $\phi \in h_{1}+H$ with $l(\phi) \leqq 3$.

Let $h_{1,1}=h_{1} ; h_{1, k}=h_{1}+h_{k}$ for $2 \leqq k$; and let $h_{k,}=h_{1}+h_{k}+h$. Observe that there are the same number of elements $\phi \in h_{1}+H$ with $l(\phi) \leqq 3$ as there are elements $\left[a_{i}, a_{j}\right]$ in the above set. Moreover observe that

$$
\operatorname{Arf}_{h_{2}, j}\left[a_{i}, a_{j}\right]=1
$$

and $\operatorname{Arf}_{h_{k, l}}\left[a_{i}, a_{j}\right]=0$ if $(k, l)<(i, j)$.

The linear independence of the set $\left\{\left[a_{1}, a_{1}\right]\right\} \cup\left\{\left[a_{i}, a_{j}\right]: 1 \leqq i<j \leqq d\right\}$ now follows easily from Lemma (5.4).

In conclusion we get the following general calculation of $W(A)$ for $A$ such that $4 A=0$.

(5.8) Theorem. (i) if $2 A=0$ then $W(A)=(\mathbb{Z} / 2)^{\left(\begin{array}{l}d \\ 2\end{array}\right)+1}$ where $d=\operatorname{rank} A$.

(ii) Suppose $2 A \neq 0$, then there is a short exact sequence

$$
0 \rightarrow(\mathbb{Z} / 2)^{\left(\begin{array}{l}
d \\
2
\end{array}\right)+1} \rightarrow W(A) \stackrel{\phi}{\longrightarrow} A \rightarrow 0
$$

that is not split. Here $d=\operatorname{rank}_{\mathbf{Z} / 2} A_{2}$.

Before the final theorem we need some preliminaries. First observe that by the above theorem $W(\mathbb{Z} / 4) \cong \mathbb{Z} / 8$. Let $B: W(\mathbb{Z} / 4) \rightarrow \mathbb{Z} / 8$ be the invariant in Brown's paper [5]. We need also to know that the inclusion $j: \mathbb{Z} / 2 \hookrightarrow \mathbb{Z} / 4$ induces a commutative diagram

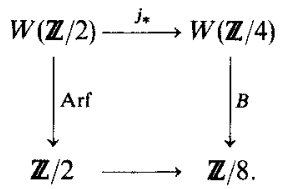

Finally, if $h: A \rightarrow \mathbb{Z} / 4$ is any map such that $h i: \mathbb{Z} / 2 \rightarrow \mathbb{Z} / 4$ is injective, define $B_{h}: W(A) \rightarrow \mathbb{Z} / 8$ by $B_{h}(V, q)=B(V, h q)$. Define

$$
B: W(A) \rightarrow \underset{h}{\oplus} \mathbb{Z} / 8
$$

to be the sum of the $B_{h}$ 's.

(5.9) Theorem. $B: W(A) \rightarrow \underset{h}{\oplus} \mathbb{Z} / 8$ is injective.

Proof. First note that $A \rightarrow \underset{h}{\oplus} \mathbb{Z} / 4$ is injective so that if $(V, q)$ is an $A$-valued form with Wu class $v \in V$ then $q_{h}(v)=0$ for all suitable $h$ implies that $q(v)=0$. Thus if $B[V, q]=0$ then by Lemma $(5.2)(V, q)$ is Witt equivalent to an $A_{2}$-valued form. So without loss of generality assume $(V, q)$ is $A_{2}$-valued. Now as $h: A \rightarrow \mathbb{Z} / 4$ runs over all allowable choices, then $h_{2}=\left.h\right|_{A_{2}}: A_{2} \rightarrow \mathbb{Z} / 4$ runs over the allowable choices $A_{2} \rightarrow \mathbb{Z} / 2$, followed by the inclusion $\mathbb{Z} / 2 \hookrightarrow \mathbb{Z} / 4$. But by the above remarks about the relation between $B$ and $\operatorname{Arf}$ we see that $\operatorname{Arf}_{h_{2}}(V, q)=0$ for all $h_{2}: A_{2} \rightarrow \mathbb{Z} / 2$. But by (5.4) we conclude that $[V, q]=0$.

\section{References}

0. Adams, J.F.: On the nonexistence of elements of Hopf invariant one. Ann. of Math. (2) 72, 20104 (1969) 
1. Barratt, M., Jones, J., Mahowald, M.: Relations amongst Toda brackets and the Kervaire invariant in dimension 62. J. London Math. Soc. (in preparation)

2. Browder, W.: The Kervaire invariant of framed manifolds and its generalizations. Ann. of Math. 90, 157-186 (1969)

3. Browder, W., Hsiang, W.C.: Some problems on homotopy theory, manifolds, and transformation groups. Proc. of Symp. in Pure Math. 32, 251-267 (1978)

4. Brown, E.H., Jr.: Kervaire invariant of a manifold. Proc. of Symp. in Pure Math. 32, 65-71 (1971)

5. Brown, E.H., Jr.: Generalizations of the Kervaire invariant. Ann. of Math. 95, 368-383 (1972)

6. Brown, E.H., Jr., Gitler, S.: A spectrum whose cohomology is a certain cyclic module over the Steenrod algebra. Topology 12, 283-295 (1973)

7. Brown, E.H., Jr., Peterson, F.P.: On the stable decomposition of $\Omega^{2} S^{r+2}$. Trans. Amer. Math. Soc. 243, 287-298 (1978)

8. Bruner, R.: An infinite family in $\pi_{*} S^{0}$ derived from Mahowald's $\eta_{j}$ family. Proc. Amer. Math. Soc. 82 (no. 4), 637-639 (1981)

9. Cohen, R.L.: Representations of Brown-Gitler spectra. Proc. Top. Symp. at Siegen, 1979. Lecture Notes of Mathematics, vol. 788, pp. 399-417. Berlin-Heidelberg-New York: Springer 1980

10. Cohen, R.L.: Stable proofs of stable splittings. Math. Proc. Cambridge Philos. Soc. 88, 149-151 (1980)

11. Cohen, R.L.: Odd primary infinite families in stable homotopy theory. Mem. Amer. Math. Soc. 242 (1981)

12. Cohen, R., Mahowald, M.: On the Adams spectral sequence of projective spaces. (in preparation)

13. Hirsch, M.W.: Immersions of manifolds. Trans. Amer. Math. Soc. 93, 242-276 (1959)

14. Kahn, D., Priddy, S.: The transfer and stable homotopy theory. Math. Proc. Cambridge Philos. Soc. 83, 103-112 (1978)

15. Kervaire, M.: A manifold which does not admit any differentiable structure. Comment. Math. Helv. 34, 256-270 (1960)

16. Lin, W.H.: Cohomology of the Steenrod Algebra. J. Pure Appl. Algebra. 29 (no. 1), 75-92 (1983)

17. Mahowald, M.: A new infinite family in ${ }_{2} \pi_{*}^{s}$. Topology 16, 249-256 (1977)

18. Mahowald, M.: Ring spectra which are Thom complexes. Duke Math. J. 46 (No. 3), 549-559 (1979)

19. Mahowald, M., Tangora, M.: Some differentials in the Adams spectral sequence. Topology 6 , 349-369 (1967)

20. Milnor, J.: The Steenrod algebra and its dual. Ann. of Math. 67, 150-171 (1958)

21. Papastavridis, S.: A formula for the obstruction to transversality. Topology 11, 415-416 (1972)

22. Snaith, V.P.: A stable decomposition for $\Omega^{n} S^{n} X$. J. London Math. Soc. 2, 577-583 (1974) 